\title{
Verunsicherung in den Mittelschichten? Konzeptionelle und methodische Erwägungen sowie empirische Befunde zur aktuellen Entwicklung in Deutschland'
}

\author{
Nicole Burzan, Silke Kohrs, Miriam Schad ${ }^{2}$
}

Technische Universität Dortmund

\begin{abstract}
Der Beitrag gibt einen Überblick über die Debatte zunehmender Statusverunsicherungen in den (deutschen) Mittelschichten. Ein Fokus liegt auf der Präsentation eigener quantitativer und qualitativer Befunde und den damit verbundenen methodischen Herausforderungen (z.B. der Operationalisierung). Quantitative Befunde zeigen, dass geäußerte ökonomische Sorgen rückläufig sind. Zugleich weisen qualitative Befunde darauf hin, dass 1) eine als unsicher empfundene Erwerbssituation nicht zwingend damit einhergeht, diese Unsicherheit prinzipiell als Bedrohung zu deuten. Andererseits können 2) Irritationen bestehen, ohne dass Sorgen explizit verbalisiert werden. Die Verschränkung von Lagemerkmalen und ggf. damit einhergehenden typischen Mentalitäten bedarf daher einer differenzierten Betrachtung.

Schlüsselwörter: Soziale Ungleichheit, Mittelschichten, Verunsicherung, Statusangst, quantitative/qualitative Methoden
\end{abstract}

\section{Einleitung}

Die Mittelschichten waren und sind in den letzten Jahren ein immer wieder präsentes Thema in der öffentlichen und wissenschaftlichen Diskussion um gesellschaftliche Entwicklungen und sozialen Zusammenhalt. Dabei werden insbesondere gestiegene Abstiegsrisiken und ängste, prekäre Lebensverhältnisse auch in mittleren Einkommenslagen oder deren Anteil an

\footnotetext{
${ }^{1}$ Die Autorinnen danken Philipp Kadelke sowie den anonymen Gutachterinnen und Gutachtern für wertvolle Hinweise zum Text.

${ }^{2}$ Nicole Burzan ist Professorin für Soziologie (Schwerpunkt soziale Ungleichheiten) an der TU Dortmund und leitet dort das Institut für Soziologie: nicole.burzan@tu-dortmund.de. Silke Kohrs ist Akademische Rätin a.Z. am Institut für Soziologie der TU Dortmund: silke.kohrs@tu-dortmund.de. Miriam Schad ist Wissenschaftliche Mitarbeiterin am Lehrstuhl für Soziologie der TU Dortmund: miriam.schad@tu-dortmund.de
}

DOI: http://dx.doi.org/10.18753/2297-8224-131 
den Wählerinnen und Wählern rechtspopulistischer Parteien thematisiert und somit problematische Trends in den Blick genommen. Umfassendere Krisendiagnosen basieren dabei auf der Annahme, dass eine Erosion der Mittelschichten (u.a. im Sinne ihrer Schrumpfung) nicht nur Auswirkungen auf die betroffenen Individuen hat, sondern dass starke Mittelschichten einen relevanten Faktor für die ökonomische und politische Stabilität von Gesellschaften darstellen (z.B. Littrell/Brooks/Ivery/Ohmer 2010; Amoranto/Chun/Deolalikar 2010; Larsen 2013; Birdsall 2015). Dabei wandeln sich (wie später noch dargestellt) die Schwerpunktsetzungen der Diskussionen sowohl in der Ungleichheitsforschung als auch in der öffentlichen Debatte um die Mittelschichten.

In unserem Beitrag werden wir Thesen zu etwaigen Verunsicherungen und Abstiegsängsten in der Mittelschicht insbesondere mit Blick auf eigene empirische Forschungen genauer beleuchten und fragen, wie verunsichert Mittelschichtangehörige sich unter welchen Bedingungen fühlen. Der Schwerpunkt unserer Argumentation liegt dabei auf der methodischkonzeptionellen Frage, welche ungleichheitsrelevanten Dimensionen mit welchen unterschiedlichen Methoden adressiert werden können. Zum einen werden wir auf der Basis des Forschungsstandes zeigen, dass unterschiedliche Befunde teilweise bereits daraus resultieren, dass es verschiedene Operationalisierungen gibt a) von sozialen Lagen, die den Mittelschichten zugeordnet werden und b) von Einstellungen, Deutungsmustern und Emotionen, die auf eine Verunsicherung und auf Statussorgen von Mittelschichtangehörigen abzielen. Zum anderen verdeutlichen wir am eigenen Material, inwiefern sich quantitative und qualitative $\mathrm{Zu}$ gänge bei ähnlichen Themen in der Konkretisierung doch insofern unterscheiden, dass sie sich gegenseitig ergänzen sowie Grenzen der Interpretierbarkeit aufzeigen können, nicht jedoch - z.B. mit Begriffen wie Sorge oder Verunsicherung - identische Phänomene erfassen.

Im Folgenden gehen wir entsprechend zunächst auf den Forschungsstand mit einem Fokus auf Operationalisierungen einer etwaigen Krise in der Mittelschicht ein. Anschließend präsentieren wir quantitative und qualitative Befunde mitsamt der angesprochenen methodischen Reflexion, bevor wir abschließend ein Fazit ziehen.

\section{Mittelschichten in der Krise}

Die Lage der Mittelschichten in Deutschland erhielt vor rund zehn Jahren eine besondere Aufmerksamkeit: Dies geschah zum einen im Kontext der Erforschung prekärer Lebensverhältnisse, welche zunehmend auch in mittleren sozialen Lagen auftraten (Castel/Dörre 2009; Vogel 2009; Kraemer 2010), zum anderen mit der These einer problematischen Schrumpfung der Mittelschichten, die in Deutschland z.B. durch das Deutsche Institut für Wirtschaftsforschung vertreten wird (Grabka/Frick 2008), nicht zuletzt gerahmt durch die globale Finanzkrise in den Jahren 2008/2009. Vor der Vergleichsfolie eines durch wirtschaftliche Prosperität gekennzeichneten golden age von den 1950ern bis in die 1970er Jahre (Schimank 2018) ließen Transformationsprozesse wie Deregulierungen der Erwerbsarbeit (auch vor dem Hintergrund von Globalisierung und Digitalisierung), ein grundlegender Umbau des Wohlfahrtsstaats nach neoliberalen Prinzipien und umfassende Individualisierungsprozesse bislang erfolgreiche Strategien des Statuserhalts sowie eine langfristige Planbarkeit des eigenen Lebens in den letzten Jahrzehnten unwägbarer erscheinen. Mit der Aufmerksamkeit für dieses Thema in der 
Wissenschaft ging eine vermehrte Aufmerksamkeit in der Öffentlichkeit einher, wenn etwa in Medienberichten Abstiegsängste von Mittelschichtangehörigen in Europa und den USA thematisiert wurden. Krisendiagnosen für die Mittelschichten bezogen sich somit zum einen auf ihre etwaige Schrumpfung, zum anderen aber auch auf Verunsicherungen Mittelschichtangehöriger, ihren Status nicht aufrechterhalten zu können oder - nicht allein auf die aktuelle Situation bezogen - ihr Leben und damit auch ihre Altersversorgung sowie den Statuserhalt ihrer Kinder schlechter planen zu können, als es den Erwartungen vorheriger Jahrzehnte entsprach (Dörre 2009).

Der Bezug auf etwaige (mittel-)schichttypische Verunsicherungen deutet darauf hin, dass soziale Lagen im Sinne verfügbarer Ressourcen immer auch mit als typisch zugeordneten Mentalitäten (Geiger 1967) bzw. entsprechendem Habitus (Bourdieu 1982) verknüpft sind, die Lageeinteilungen erst über sozialstatistische Kategorien hinaus sinnvoll werden lassen. Der Mittelschicht wurden z.B. über längere Zeit Leistungswille, individuelles Aufstiegsstreben, rationale Alltagsgestaltung und langfristige Planung (Hradil/Schmidt 2007; Schimank/Mau/Groh-Samberg 2014) als typisch zugeschrieben. Mit der Infragestellung bislang oft erfolgreicher Strategien des Statuserhalts zeigt sich allerdings, dass das Wechselverhältnis zwischen Lagen und kulturellen Orientierungen ein höchst dynamisches ist. So sind Mentalitäten nicht mechanisch als Folge ressourcenbedingter Lagen $\mathrm{zu}$ verstehen, sondern als wichtiges eigenständiges Element sozialer Ungleichheiten zu begreifen, insofern beispielsweise vermehrt geäußerte Verunsicherungen nicht zwangsläufig konkret erhöhte Abstiegsrisiken wiederspiegeln.

In welchem Ausmaß Mittelschichtangehörige verunsichert sind und wie sich diese Verunsicherungen entwickelt haben, ist in der Forschung umstritten. Unsere These lautet, dass dies nicht zuletzt auf variierende methodische Zugänge und Perspektivierungen sowie auf unterschiedliche Operationalisierungen von a) mittleren sozialen Lagen und b) Statusverunsicherung in der quantitativen und qualitativen Forschung zurückzuführen ist.

Ad a): Bereits die Rede von der Mittelschicht oder den Mittelschichten stellt dabei im Vergleich zu Begriffen wie Klasse oder Milieu eine gewisse konzeptionelle Vorentscheidung dar. Mittlere Lagen gelten seit jeher als heterogen und werden daher in der Forschung häufig weiter vertikal und/oder horizontal unterteilt. Dies gilt bereits für das in den 1960er Jahren prominente Modell der sogenannten Zwiebel nach Bolte et al. (1967). In soziologischen Ansätzen spielen dabei neben dem Einkommen oft weitere Merkmale wie der Beruf oder die Bildung eine wichtige Rolle, zudem - gerade für horizontale Differenzierungen - z.B. die Verfügung über mehr oder weniger ökonomisches oder kulturelles Kapital (Bourdieu 1982) oder milieuspezifische Wertorientierungen (z.B. bei Vester/Oertzen/Geiling/Hermann/Müller 2001, Reckwitz 2017). Schimank et al. (2014; Schimank 2015) gehen sogar davon aus, dass es eine «investive Statusarbeit» (Groh-Samberg/Mau/Schimank 2014: 223) ist, die mittlere Lagen charakterisiert, denn deren Angehörige können zwar nicht auf sorgenfreien Wohlstand bauen, verfügen andererseits aber über so viele Ressourcen, dass sie etwas zu verlieren haben und Statusinvestitionen möglich sind. Generalisierte Aussagen über die Mittelschicht(en) sind daher kaum möglich und rufen bei Rezipient/innen entsprechend unterschiedlichste Assoziationen sozialer Lagen (von Facharbeiter/innen bis zu Akademiker/innen) hervor, was in der Fol- 
ge die Vergleichbarkeit der Zustandsbeschreibungen und Diagnosen innerhalb der Diskussion erschwert.

Wie sich der Anteil der Mittelschichten im Schichtgefüge insgesamt und wie sich entsprechende Auf- und Abstiege entwickelt haben, ist folglich davon abhängig, wie man diese operationalisiert. Quantitative Analysen, die oft auf dem Einkommen als Schichtkriterium beruhen, stellen dabei im europäischen Vergleich eine Schrumpfung der Mittelschicht fest, die jedoch für Deutschland nicht dramatisch ausfällt (zu globalen Mittelschichten Popp 2015). So konstatieren Grabka/Goebel/Schröder/Schupp (2016) für die deutschen Mittelschichten einen Rückgang zwischen 1991 und 2013 von 66 auf 61 Prozent. Trends in diese Richtung beobachten auch Horn/Behringer/Gechert/Rietzler/Stein (2017) und Grabka/Westermeier (2014), während Arndt (2015) zeigt, dass es von 2006 bis 2012 nur geringe Veränderungen des Bevölkerungsanteils in der Einkommensmitte gegeben habe. Dies bestätigt auch Niehues (2017). Verwiebe (2014) stellt ein Schrumpfen der Mittelschicht 1991 bis 2012 insbesondere für die migrantische Bevölkerung in Deutschland fest. Daten des Pew Research Centers zeigen, dass es in Deutschland wie in einigen anderen (z.B. USA, Spanien, Norwegen), aber nicht allen der betrachteten Länder (z.B. nicht in Frankreich oder Großbritannien) zwischen 1991 und 2010 eine gewisse Abnahme des Mittelschichtanteils gegeben hat (in Deutschland nach diesem Messkonzept von 78 auf 72 Prozent, Kochhar 2017: 4). Diese Befunde wiederum können zu unterschiedlichen Schlussfolgerungen führen. So wäre es etwa unzutreffend anzunehmen, die Schrumpfung bedeute allein zunehmende Abstiege in untere soziale Lagen. Vielmehr ist die Differenz laut Kochhar (2017: 20) zwischen der Anteilsveränderung im Segment der höheren Einkommen und der niedrigeren Einkommen positiv, d.h. in Deutschland und den meisten anderen Ländern gab es mehr Auf- als Abstiege (bzw. war die Abnahme in den unteren Einkommensgruppen höher als in den oberen). Konträre Befunde sind demgegenüber bei Hartmann (2018: 122) zu finden, demzufolge die Abstiege die Aufstiege überwiegen. Jedenfalls gibt es privilegierte soziale Lagen am oberen Ende der Sozialstruktur, die von den Entwicklungen profitieren können, während sich Armutslagen tendenziell verfestigen. Diese Befunde bestätigen die Diagnosen zu neuen Dynamiken sozialer Ungleichheit innerhalb vieler Länder (GrohSamberg/Hurch/Waitkus 2018). Auf der Ebene subjektiver Schichtzuordnungen fühlen sich weiterhin viele Befragte in Surveys der Mittelschicht zugehörig. ${ }^{3}$ Auf der Basis bestehender Studien gehen wir im vorliegenden Beitrag folglich von Mittelschichten aus, die, ökonomisch operationalisiert, einer gewissen Schrumpfung unterliegen, was jedoch nicht mit erhöhten Abstiegsrisiken per se gleichzusetzen ist. Einige Autor/innen (z.B. Mau 2012) weisen allerdings darauf hin, dass auch ein geringes Ausmaß an Schrumpfung mit einer Erosion bestehender Vorstellungen über die Lage und die Sicherheit in den Mittelschichten einhergehen kann.

Ad b): Auch bei dem zweiten Punkt, der Diskussion um die Verunsicherung und geäußerte Sorgen, wird bei näherer Betrachtung deutlich, dass hier ganz unterschiedliche Dimensionen gemeint sein können. Neben den Sorgen einer Person in bestimmten Lebensbereichen tauchen beispielsweise die Begriffe Statusangst und Statusunbehagen (status anxiety) bzw. Statusunsicherheit (status insecurity) in der Ungleichheitsforschung auf. Bekannt geworden

\footnotetext{
${ }^{3}$ Laut Datenreport 2018: 260 waren es 2016 in Westdeutschland 60 und in Ostdeutschland 54 Prozent; die alternativen Antworten waren: obere Mittelschicht/Oberschicht, Arbeiter- und Unterschicht.
} 
sind diese Begrifflichkeiten nicht zuletzt durch die Spirit-Level-Theorie von Wilkinson und Pickett (2010), die das Statusunbehagen in ungleichen Gesellschaften als einen zentralen Mechanismus beschreiben, der unter anderem zu einer geringeren Lebenszufriedenheit führt. Empirisch operationalisiert wird hierbei dieses relationale Statusunbehagen über den Vergleich zu anderen und insbesondere die Wahrnehmung durch andere. ${ }^{4}$ Andere Autor/innen definieren die Statusangst ${ }^{5}$ als „Angsterscheinungen, die bei tatsächlichem oder erwartetem Statuswechsel auftreten, entweder aufgrund eines verbundenen Prestigeverlusts oder auch aus Angst vor den mit dem neuen Status verbundenen Anforderungen“ (Laatz 2011: 654). Die Statusunsicherheit (in Abgrenzung zum Statusunbehagen) ist bei Delhey und Dragolov (2014: 154) wiederum durch die Angst vor einer Abwärtsmobilität gekennzeichnet. Des Weiteren finden sich auch Begriffe wie Abstiegsangst (z.B. Nachtwey 2016) oder Statuspanik (Bude 2011), die stellenweise nicht genauer expliziert werden und weiterer Klärung bedürften. Als Schwierigkeit begrifflicher Trennschärfe kommt hinzu, dass die benutzten Termini teilweise synonym verwendet werden. ${ }^{6}$

Zum einen erschweren also konzeptionelle Variationen eine konsensuelle empirische Diagnose von Verunsicherungen in den Mittelschichten. Zum anderen hat sich der Schwerpunkt des Diskurses verlagert - von der Diagnose einer möglichen Erosion der Mittelschichten und den individuellen Folgen für Mittelschichtangehörige auf die Einbettung dieser Dimensionen in makrogesellschaftliche Phänomene. So geht es aktuell in diesem Kontext vermehrt um globale soziale Ungleichheiten (z.B. Milanović 2016; Weiß 2017), somit auch um innerhalb vieler Länder wachsende soziale Ungleichheiten (Groh-Samberg et al. 2018) sowie um die Mobilisierbarkeit sozialer Lagen für rechtspopulistische Tendenzen (z.B. Koppetsch 2018b; Schimank 2018) und um Abgrenzungspraktiken und soziale Schließungen (Schöneck/Ritter 2018). Krisenhafte Momente und kritische Perspektiven bleiben im Blick auf die Mittelschichten dabei prinzipiell erhalten, wenn z.B. die wachsenden globalen Mittelschichten in manchen Regionen als vergleichsweise vulnerabel charakterisiert werden. Dabei wird auch grundsätzlich hinterfragt wird, ob sich bestehende Konzeptionen von Mittelschichten etwa auf den globalen Süden übertragen lassen (Davis 2004; Popp 2015; Kroeker/O'Kane/Scharrer 2018). Zudem wird in verschiedenen Ländern das Potenzial für politisch extreme Haltungen und insbesondere rechtspopulistische Orientierungen auch in (einigen) mittleren sozialen Lagen aktuell verstärkt beobachtet (für Deutschland: Decker et al. 2016; Zick et al. 2016; Vester 2017; für Europa und die USA: Kriesi/Grande/Lachat/Dolezal 2006; Hochschild 2016; Inglehart/Norris 2016). Extreme Orientierungen in mittleren Lagen sind historisch indessen keine neue Erscheinung (vgl. etwa Geiger 1967; Parkinson 1968; Johnston 2013).

Wie sich Verunsicherung in Deutschland empirisch darstellt und welche Rolle dabei unterschiedliche methodische Zugänge spielen, zeigen nun die folgenden Abschnitte.

\footnotetext{
${ }^{4}$ So operationalisieren Delhey (2012) sowie Delhey und Dragolov (2014) das Statusunbehagen über die folgenden Variablen des European Quality of Life Survey (EQLS): a. Ich habe nicht das Gefühl, dass die Leute in meiner Umgebung den Wert dessen, was ich tue, anerkennen. b. Einige Leute sehen wegen meiner Arbeit oder meines Einkommens auf mich herab.

${ }^{5}$ Auch hier wird der Begriff der status anxiety verwendet.

${ }^{6}$ Beispielsweise operationalisieren Lengfeld und Ordemann (2017) Abstiegsängste als Sorgen um den Verlust des Arbeitsplatzes.
} 


\section{Verunsicherung in der Mittelschicht - quantitative Befunde}

Viele Untersuchungen zu Mittelschichten in Deutschland greifen sekundäranalytisch auf das sozioökonomische Panel (SOEP) zurück, eine seit 1984 durchgeführte, nahezu bevölkerungsrepräsentative jährliche Mehrthemenumfrage (Wagner/Frick/Schupp 2007). Die Schichtzugehörigkeit wird dabei - auch mit schichtinternen Differenzierungen - unterschiedlich operationalisiert, etwa auf der Basis des Einkommens (z.B. Goebel/Gornig/Häußermann 2010; Werding 2011; Verwiebe 2014), des Vermögens (z.B. Arndt 2015) oder auf der Basis von Merkmalen wie der beruflichen Stellung und der Bildung. So wird etwa das EGP-Klassenschema verwendet (z.B. Burzan/Kohrs/Küsters 2014; Lengfeld/Ordemann 2016, jeweils mit unterschiedlicher Schichtzuordnung der Klassen) oder eine Kombination aus Bildung, Beruf und Einkommen (Burkhardt/Grabka/Groh-Samberg/Lott/Mau 2013; in einem zweistufigen Verfahren: Niehues/Schaefer/Schröder 2013; Niehues/Orth 2018). Auch die Untersuchung der Verunsicherung stützt sich jeweils auf unterschiedliche Items. Häufig gewählte Messkonzepte nutzen (große) Sorgen um die eigene wirtschaftliche Situation (Burzan et al. 2014; Burkhardt et al. 2013; Niehues/Orth 2018), oder (einige und große) Sorgen um den Verlust des Arbeitsplatzes (auf Basis von Daten des Wohlfahrtssurvey und des ALLBUS Böhnke 2005; auf der Basis von SOEP-Daten Lengfeld/Hirschle 2009; Lengfeld/Ordemann 2017).

Krisendiagnosen zur Lage der Mittelschichten wurden dabei zunächst insbesondere durch den Blick auf Verläufe gestützt, die sich auf den Zeitraum von den 1990ern bis zur Mitte der 2000er Jahre beziehen. Die Befunde der genannten Studien gingen in die gleiche Richtung: Es wurden (auch) in mittleren Lagen in zunehmendem Maße (große) Sorgen um die eigene wirtschaftliche Situation oder den Verlust des Arbeitsplatzes geäußert. Untersuchungen zum weiteren Verlauf zeigten jedoch, dass sich dieser Trend einer hohen bzw. steigenden Verunsicherung nicht fortsetzte (Burkhardt et al. 2013; Burzan et al. 2014; Lengfeld/Ordemann 2017): Ab der Mitte des Jahrzehnts ging der Anteil derjenigen, die sich (große) Sorgen um ihre wirtschaftliche Situation oder den Verlust ihres Arbeitsplatzes machten, zurück. Schöneck/Mau/Schupp (2011) zeigten allerdings, dass Sorgen in Bezug auf langfristige potentielle Abstiegsgefährdungen weiterhin bedeutsam waren.

Anhand eigener Befunde einer früheren Studie (Burzan et al. 2014) mit Daten des SOEP bis 2011, die wir nun mit aktuellen Umfragedaten (Welle SOEP v33) erweitern können, werfen wir im Folgenden einen differenzierten Blick auf die Entwicklung bis einschließlich 2016. Dabei erläutern wir zunächst unsere spezifische Operationalisierung der Schichtzugehörigkeit und der Verunsicherung, bevor wir Aussagen zu neueren Trends machen.

Die Schichtzugehörigkeit haben wir anhand des EGP-Klassenschemas (Erikson/Goldthorpe 1992) operationalisiert und dabei alle Erwerbstätigen zwischen 18 bis 65 Jahren in der Stichprobe nach Unter-, Mittel- und Oberschicht differenziert (s. Tabelle 1). Die sieben Klassen (mit jeweiligen Unterklassen) beinhalten die berufliche Stellung (u.a. das Ausmaß an Autonomiebefugnis) und die internationale Standardklassifikation von Berufen (ISCO). Berufszugehörigkeiten bilden dabei unseres Erachtens schichttypische Mentalitäten besser $\mathrm{ab}$ als eine rein einkommensbasierte Schichteinteilung. 
Tabelle 1: Schichtzuordnung nach dem EGP-Klassenschema

\begin{tabular}{|c|c|c|c|c|}
\hline & EGP-Klassen & & deutsche Bezeichnung & Zuordnung \\
\hline \multirow{2}{*}{$\begin{array}{l}\text { Dienst- } \\
\text { Verhältnis }\end{array}$} & High service & $\mathrm{I}$ & Obere Dienstklasse & Oberschicht \\
\hline & Low service & II & Untere Dienstklasse & $\begin{array}{l}\text { Mittelschicht } \\
\text { (eng) }\end{array}$ \\
\hline \multirow[t]{4}{*}{$\begin{array}{l}\text { Zwischen- } \\
\text { stellung }\end{array}$} & Routine non-manual & IIIa & Gehobene Routinetätigkeit & $\begin{array}{l}\text { Mittelschicht } \\
\text { (weit) }\end{array}$ \\
\hline & Routine service-sales & IIIb & $\begin{array}{l}\text { Untere Routinetätigkeit (in Service und } \\
\text { Verkauf) }\end{array}$ & Unterschicht \\
\hline & $\begin{array}{l}\text { Self employed with } \\
\text { employees }\end{array}$ & IV & Selbständige mit Mitarbeitern & Mittelschicht \\
\hline & $\begin{array}{l}\text { Self employed without } \\
\text { employees }\end{array}$ & IV & Selbständige ohne Mitarbeiter & Mittelschicht \\
\hline \multirow{2}{*}{$\begin{array}{l}\text { Lohn- } \\
\text { Arbeitsver- } \\
\text { hältnis }\end{array}$} & Skilled manual & $\mathrm{V}-\mathrm{VI}$ & Facharbeiter & Unterschicht \\
\hline & Semi-unskilled manual & VII & Un- und angelernte Arbeiter & Unterschicht \\
\hline
\end{tabular}

EGP-Klassenschema nach Erikson/Goldthorpe 1992: 38f.; eigene Darstellung; nur Erwerbstätige

Weiterhin haben wir die Mittelschichten hier eng gefasst als erwerbstätige Angehörige der unteren Dienstklasse sowie Selbständige (bzw. in einer erweiterten Variante zusätzlich Beschäftigte in gehobenen Routinetätigkeiten). Der Grund dafür besteht darin, dass die These der Verunsicherung dadurch gut überprüfbar ist: Während Unsicherheiten für Angehörige einer unteren Mittelschicht vermutlich keinen großen Neuheitswert haben, würden erhöhte Anteile sich Sorgender in der hier konzipierten Mittelschicht deutlich auf die Bestätigung der Krisendiagnose hindeuten. Untere Schichten sind entsprechend nicht als (randständige) Armutslagen $\mathrm{zu}$ interpretieren. Wir folgen damit einer historisch hergeleiteten Differenzierung zwischen qualifizierten Angestellten und Selbständigen einerseits und gering qualifizierten Angestellten sowie Arbeiter/innen andererseits.

Zur Erfassung von Verunsicherungen wählen wir den Anteil derjenigen, die sich große Sorgen um ihre wirtschaftliche Situation machen. Der Vorteil dieses Indikators ist, dass er im Unterschied zur Sorge um den Arbeitsplatz den Haushaltskontext mit einbezieht. Für die Beantwortung der Frage nach den Sorgen stehen im SOEP-Fragebogen die Möglichkeiten "große“, „einige“ und „keine Sorgen“ zur Verfügung (Abbildung 1); für die verschiedenen Vergleiche ziehen wir den Anteil derjenigen heran, die angegeben haben, sich große Sorgen zu machen. 


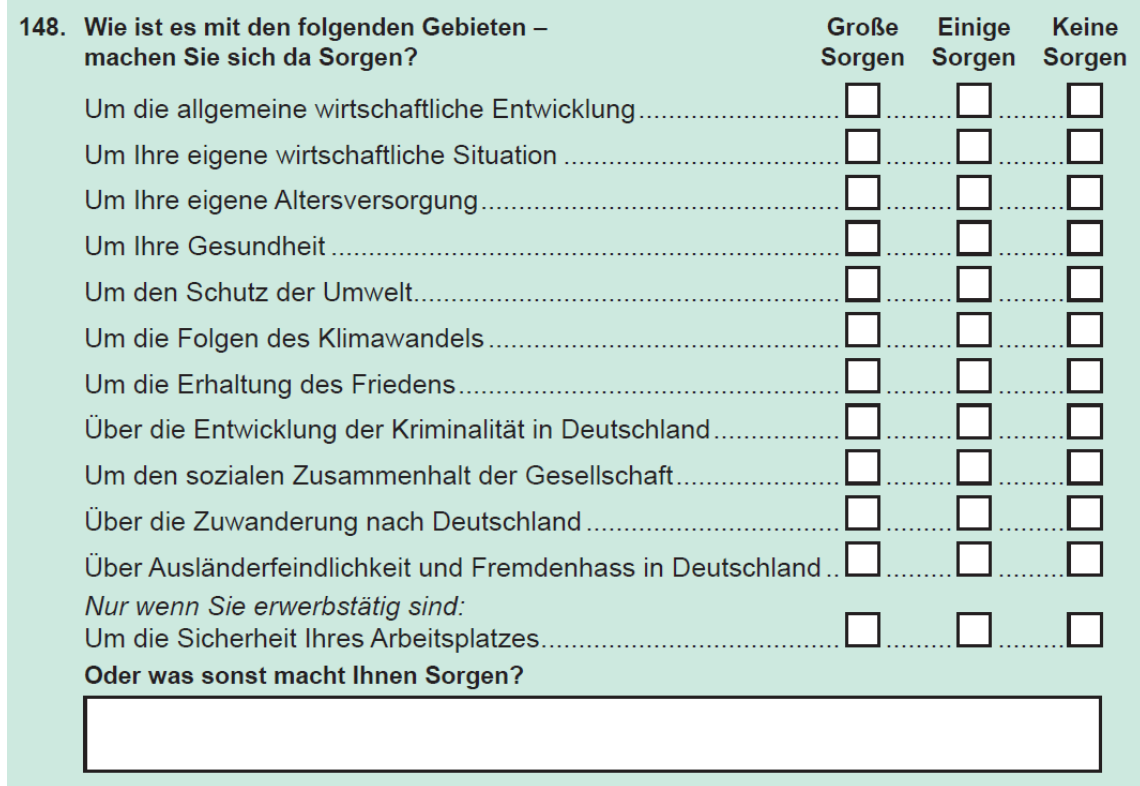

Abbildung 1: Fragebogenfrage „Sorgen“(DIW SOEP-Personenfragebogen 2016)

Abbildung 2 gibt zunächst einen Überblick über die Befunde im Schicht- und Zeitvergleich:

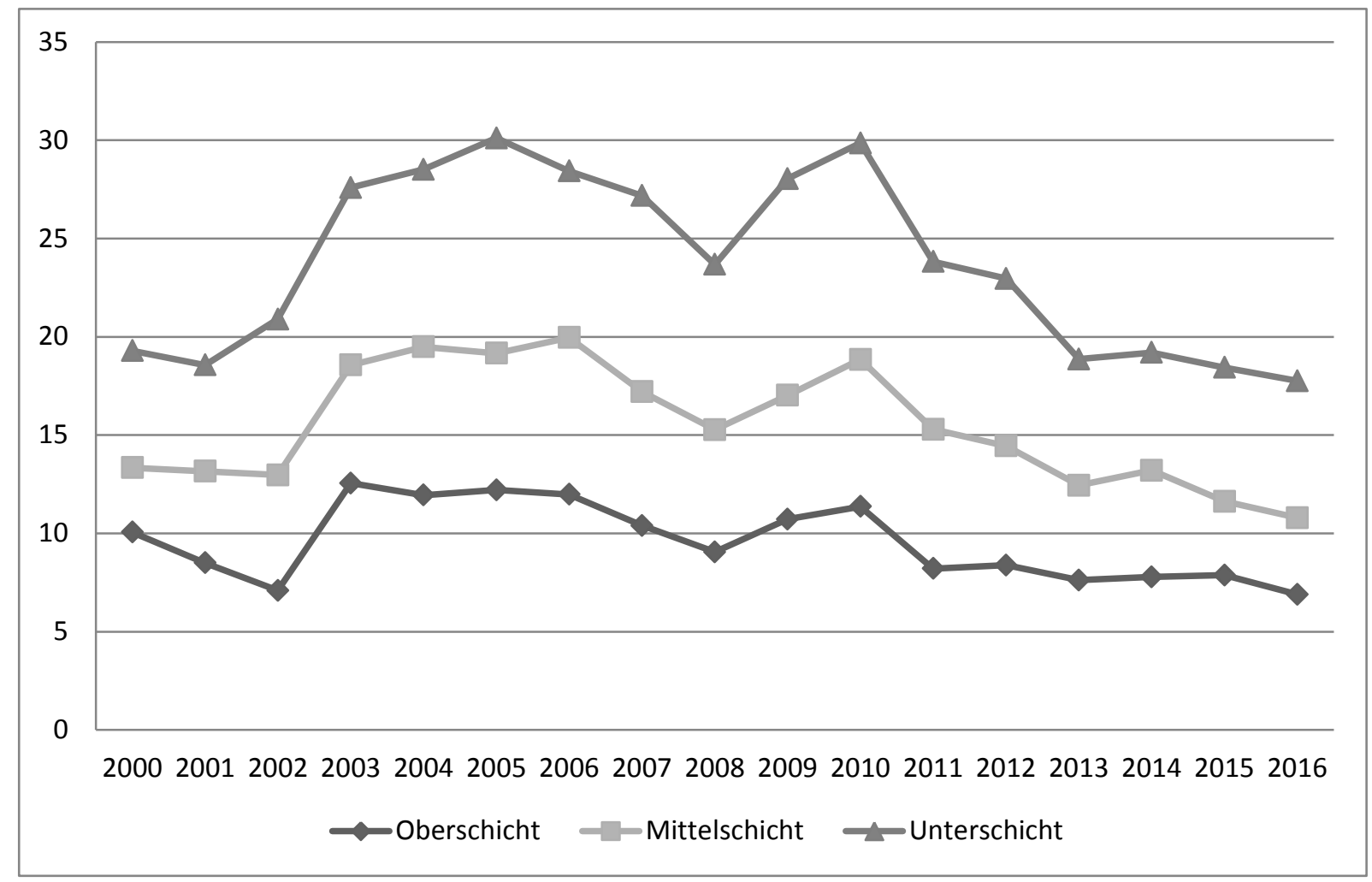

Abbildung 2: Anteile großer Sorgen um die wirtschaftliche Situation im Schichtvergleich. Anmerkung: Sozioökonomisches Panel (SOEP), Daten für die Jahre 1984-2016 (SOEP v33), N= 219.278 (Beobachtungen), eigene Berechnungen, nur Erwerbstätige 
Die Graphiken zeigen, dass der Anteil der Mittelschichtangehörigen mit großen Sorgen von 2002 bis 2006 anstieg, während es 2006 in den beiden anderen Schichten einen Rückgang gab. Dieser Trend setzte sich allerdings nicht fort, sondern es lässt sich ein Rückgang beobachten. Unterbrochen wurde dieser Rückgang kurzzeitig 2009 und 2010, da sich hier zeitversetzt die Finanzkrise und die öffentliche Debatte um erodierende Mittelschichten auswirkten. Seit 2010 gibt es einen weiteren Rückgang. Vergleicht man den Gesamtzeitraum von 2000 bis 2016, zeigt sich, dass im Jahr 2016 sogar weniger Personen in der Mittelschicht verunsichert waren als im Ausgangsjahr 2000: 2016 machten sich knapp 11 Prozent große Sorgen gegenüber rund 13 Prozent im Jahr 2000. ${ }^{7}$

Zählt man in einem erweiterten Konzept Beschäftigte in gehobenen Routinetätigkeiten (s.o. Tabelle 1) zu den Mittelschichten hinzu, ergeben sich keine nennenswerten Veränderungen. Angehörige der in diesem Sinne weiteren Mittelschichten machen sich zu einem geringfügig erhöhten Anteil große Sorgen um ihre wirtschaftliche Situation; auch die Entwicklung im Zeitverlauf ist nahezu identisch.

Der gleiche Trend auf etwas niedrigerem Niveau und mit weniger großen Veränderungen ist für die große Sorge um den Verlust des Arbeitsplatzes im Zeitverlauf zu beobachten. Insgesamt sind die Arbeitsplatzsorgen in allen drei Schichten geringer als die Sorge um die eigene wirtschaftliche Situation und relativ ähnlich in der Entwicklung. Die Veränderungen in der oberen Schicht sind am geringsten: zwischen 2011 und 2015 auf nahezu gleich hohem Niveau, fallen die Sorgen 2016 auf gerade einmal 4 Prozent. Parallel zu der Arbeitslosenquote in Deutschland, die kontinuierlich von 2011 (7,9 Prozent) bis 2016 (6,8 Prozent) sinkt (Statistik der Bundesagentur für Arbeit 2017), sorgen sich auch in den Mittelschichten immer weniger Erwerbstätige um ihren Arbeitsplatz (7,4 Prozent im Jahr 2011 und 5,9 Prozent im Jahr 2016). Auffällig ist für alle hier angesprochenen Verläufe, dass die Mittelschichten sich stets in mittlerem Maße Sorgen machen und die Entwicklung in den drei Schichten jeweils relativ ähnlich verläuft. Dies deutet auf Einflüsse bestimmter Entwicklungen auf der Makroebene hin (z.B. Finanzkrise, Rückgang der Arbeitslosenquote) und weniger auf spezifische Mentalitäten, aufgrund derer Angehörige unterschiedlicher Schichten variierend auf ähnliche Entwicklungen reagieren würden. Andererseits bilden sich allgemeine Entwicklungen wie die Deregulierung der Erwerbsarbeit vor dem Hintergrund von Globalisierung, Digitalisierung oder der Diskussion um Migrationsprozesse nicht in einem stabil höheren Niveau kontextunspezifisch geäußerter Unsicherheit um die eigene wirtschaftliche Situation oder den Verlust des Arbeitsplatzes ab. Dies liegt nicht daran, dass insgesamt ein höheres Niveau an Gelassenheit oder sinkende Sicherheitserwartungen zu verzeichnen wären. So weisen im Vergleich der kumulierten Zeiträume 2008-2010 und 2014-2016 einzelne Bereiche ein deutlich höheres Niveau auf (z.B. die Sorge um die Kriminalitätsentwicklung in Deutschland), in anderen Bereichen stiegen die Sorgen sogar deutlich an: z.B. große Sorgen um den Erhalt des Weltfriedens, um Fremdenhass in Deutschland oder um das Thema der Zuwanderung (s. Abbildung 3).

\footnotetext{
${ }^{7}$ Damit sind die Werte im Jahr 2016 in der Mittelschicht auch unter dem Niveau von 1984 (14,9 Prozent in Westdeutschland).
} 


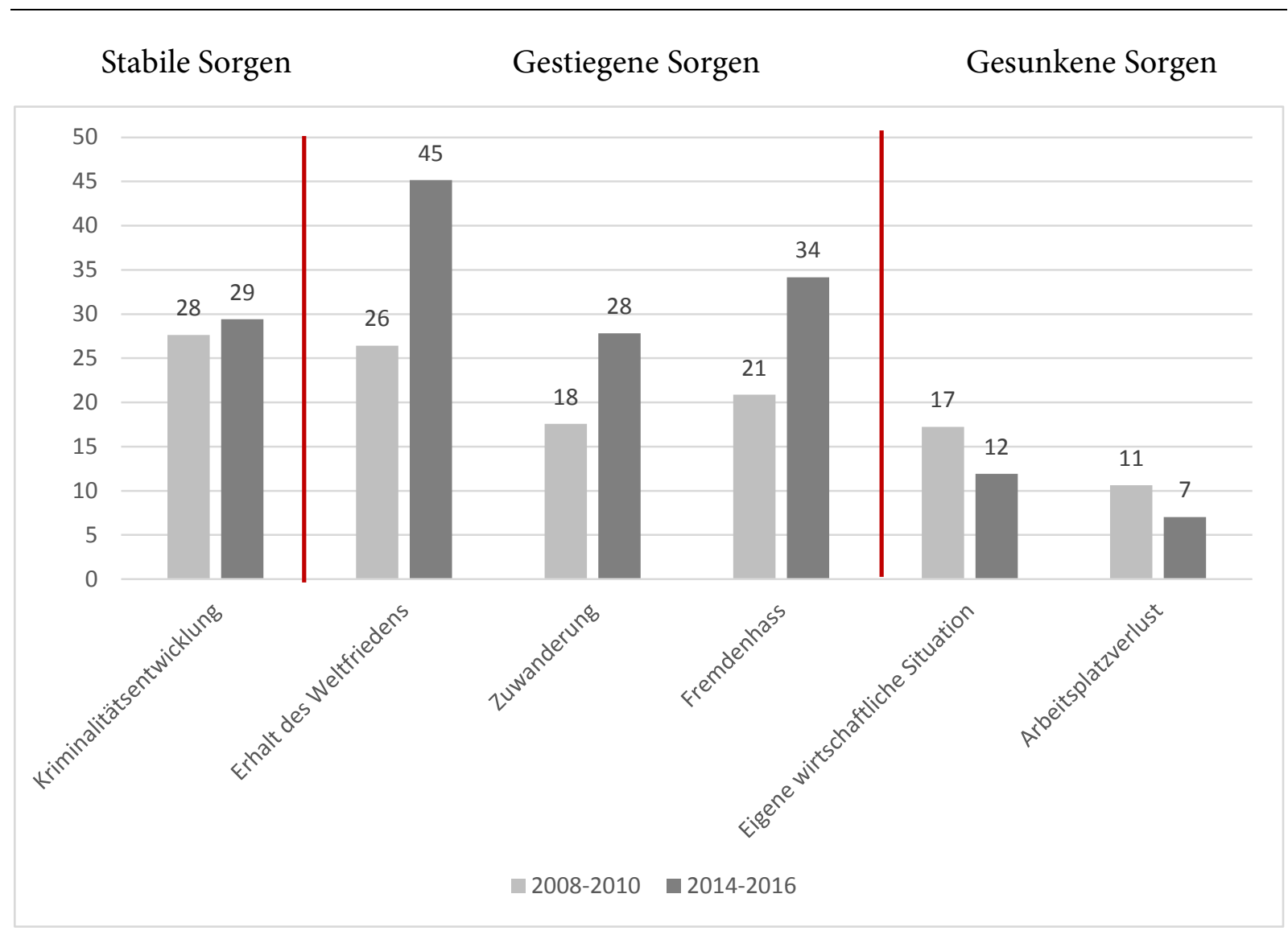

Abbildung 3: Kumulierte Anteile großer Sorgen in der Mittelschicht im Vergleich. Anmerkung: Sozioökonomisches Panel (SOEP), Daten für die Jahre 1984-2016 (SOEP v33), eigene Berechnungen, nur Erwerbstätige.

Wirft man einen Blick auf Gemeinsamkeiten und Unterschiede der großen Sorge - nun wiederum um die wirtschaftliche Situation - innerhalb der Mittelschichten, so zeigen sich auch hier differenzierende Faktoren, u.a. bezogen auf die Lebensform, die Region oder erwerbsbezogene Merkmale. Viele der bereits in der Vorgängerstudie (mit Daten bis 2011; vgl. Burzan et al. 2014) herausgearbeiteten Zusammenhänge innerhalb der Mittelschichten lassen sich bivariat auch für die jüngeren Jahre bestätigen: ${ }^{8}$ So wirkt sich eine Partnerschaft nach wie vor sorgenreduzierend aus, und es gibt weiterhin mehr sich sorgende Erwerbstätige in Ost- als in Westdeutschland. ${ }^{9}$ Ebenfalls nahezu unverändert sind die erwerbsbezogenen Merkmale Arbeitslosigkeitserfahrung und Befristung der Tätigkeit (beide sorgenverstärkend) sowie Vollzeit- vs. Teilzeitbeschäftigung (nach wie vor kein Effekt). Frauen machten sich in den letzten Jahren häufiger große Sorgen als Männer (während es zuvor keinen signifikanten $\mathrm{Zu}$ sammenhang gab); die Anteile verschiedener Altersgruppen nähern sich tendenziell an.

Ein wesentlicher Befund unserer Untersuchung besteht mithin darin, dass trotz des beobachtbaren Rückgangs des Anteils derjenigen mit großen Sorgen um die eigene wirtschaftli-

\footnotetext{
${ }^{8}$ Bei den hier aufgeführten Analysen handelt es sich um bivariate Zusammenhänge zwischen der großen Sorge um die eigene wirtschaftliche Situation und dem jeweils aufgeführten Merkmal für den Zeitraum 2006 bis 2016, d.h. für die Phase der rückläufigen Sorgen.

${ }^{9}$ Ihre Anteile haben sich allerdings angenähert.
} 
che Lage die Mittelschichten nach wie vor eine heterogene Gruppierung mit nennenswerten internen Differenzierungen darstellen. Der klare Trend des Sorgerückgangs ist also nicht mit homogeneren Perspektiven innerhalb der Mittelschichten verknüpft.

Von einem Rückgang der Verunsicherung im Sinne der genannten Indikatoren innerhalb der Mittelschichten gehen auch die zuvor genannten Studien aus. Exemplarisch sei die Studie von Lengfeld und Ordemann (2017, 2018 jeweils mit Daten bis 2014) genannt, die die Mittelschichten zwar ebenfalls mit Hilfe des EGP-Klassenschemas identifizieren, jedoch eine andere Zuordnung vornehmen: Angehörige der unteren Dienstklasse bilden dort die obere, gehobene Routineangestellte die mittlere und Techniker/Facharbeiter die untere Mittelschicht (Lengfeld/Ordemann 2017: 173). Als abhängige Variable verwenden sie die Sorgen um den Verlust des Arbeitsplatzes - einige Sorgen reichen hier allerdings als Indikator für Verunsicherung bereits aus. Trotz der unterschiedlichen Operationalisierung zeigt sich: Auch nach diesem empirischen Konzept ist ein deutlicher Rückgang der Sorgen nach 2007 zu verzeichnen. ${ }^{10}$

Angesichts zum Teil alarmierend formulierter Krisendiagnosen kann man mit Hilfe dieser und anderer Studien zusammengefasst eine gewisse Entwarnung im Hinblick auf die subjektive ökonomische Verunsicherung der Mittelschicht geben: Mittelschichtangehörige machen sich im Schichtvergleich in mittlerem Maße Sorgen um ihre wirtschaftliche Situation und tendenziell nimmt das Ausmaß der Sorgen im Zeitverlauf ab. Allerdings sind durchaus nennenswerte Differenzierungen innerhalb der Mittelschichten zu konstatieren. Eine völlige Entwarnung ist also nicht zu geben, nicht nur angesichts dieser Differenzierungen, auch angesichts bestimmter methodischer Spezifika: So konnten wir einerseits mit dem SOEP auf bevölkerungsrepräsentative Daten mit hohen Fallzahlen (auch innerhalb der Schichten) zugreifen, die differenzierte Analysen sowohl im Schicht- als auch im Zeitvergleich ermöglichen. Andererseits haben wir hier eine recht eng gefasste Mittelschicht qualifizierter Erwerbstätiger betrachtet und waren dabei auf Indikatoren für Verunsicherung aus dem vorliegenden Datensatz angewiesen. Insbesondere die grobe Erfassung der Antwortkategorien für Indikatoren der Verunsicherung (große, einige, keine Sorgen) stellte eine Einschränkung für unsere Analysen dar. Weiterhin werden die Einstellungen in einem bestimmten Kontext erhoben (hier: Sorgen in unterschiedlichen Lebensbereichen), was zwar einerseits den Vergleich aufgeführter Sorgenbereiche ermöglicht, andererseits jedoch wenig Raum z.B. für latente Verunsicherungen und Irritationen lässt. In der vorliegenden Analyse haben wir uns zudem in Bezug auf den zeitlichen Horizont mit den verwendeten Indikatoren v.a. auf Sorgen um die kurz- und mittelfristige wirtschaftliche Situation bezogen. Der Anteil derjenigen mit großen Sorgen um die - erst ab 2015 im SOEP erfragte - eigene Altersversorgung etwa liegt mit 23 Prozent auf einem deutlich höheren Niveau als große Sorgen um die eigene wirtschaftliche Situation (12 Prozent). ${ }^{11}$ Was die Angabe großer Sorgen um die eigene wirtschaftliche Situation weiter bedeutet, z.B. für Statusängste, die eher kulturelle Hegemonieansprüche betreffen, oder für Kon-

\footnotetext{
${ }^{10}$ Allerdings teilen wir die Schlussfolgerung nicht, dass gerade die mittlere Mitte das in Bezug auf Verunsicherungen einstellungssensible Zentrum der Gesellschaft sei. Nicht nur ist die gewählte Hierarchisierung des EGP-Klassenschemas aus unserer Sicht diskussionswürdig, sondern empirische Unstimmigkeiten werden von den Autor/innen selbst genannt, ohne dass sie ihre These dadurch relativieren (z.B. die fehlende Reaktion dieses Schichtsegments auf die Finanz- und Wirtschaftskrise; Lengfeld/Ordemann 2017: 182).

${ }^{11}$ Durchschnitt der Werte für 2015-2016; ähnlich auch Schöneck et al. (2011) auf der Basis des Items „Wahrscheinlichkeit, den gegenwärtigen Lebensstandard im Alter beibehalten zu können“.
} 
sequenzen auf der Handlungsebene, kann mit den vorliegenden Daten allenfalls begrenzt (über Korrelationen) erfasst werden. Die Postulierung von Kausalzusammenhängen und komplexen Konstellationen im Zusammenhang mit (Un-)Sicherheit bleibt dabei jedoch schwierig (vgl. dazu genauer Burzan et al. 2014: 97). Infrage steht somit nicht allein, welchen Aspekt von Verunsicherung man mit der Erhebung entsprechender Items in Surveys erfasst, sondern auch, ob man die Mittelschichten betreffende Problematiken im weiteren Kontext von Statuskonflikten dadurch bereits hinreichend adressiert. Wir fragen daher im nächsten Schritt danach, welchen Beitrag qualitative Untersuchungen ggf. zur Beantwortung dieser blinden Flecken leisten können und wo umgekehrt die Grenzen dieser Forschungsdesigns liegen, um Verunsicherungen in den Mittelschichten zu erfassen.

\section{Verunsicherung in der Mittelschicht - qualitative Befunde}

Der Blick auf nuancierte Abstufungen von Verunsicherungen und ihre Kontexte stellt eine Stärke qualitativer Untersuchungen dar, die sich mit dem breiten Spektrum möglicher Erosionen oder Statusunsicherheiten in den Mittelschichten beschäftigen. Dabei steht selten die gesamte Mitte im Mittelpunkt des Forschungsinteresses. Die qualitativen Samples variieren deutlich und betrachten ausgewählte Teilgruppen, die den Mittelschichten zugerechnet werden oder als Repräsentanten der Mitte oder einzelner Segmente fungieren. Eine Reihe qualitativer Studien wirft dabei einen Blick auf die Umgangsweisen und Bewältigungsstrategien von Mittelschichtangehörigen, bei denen man von einer Verunsicherung ausgeht. Dabei ermöglichen diese Studien nicht nur, nach spezifischen Handlungen zu fragen (z.B. nach dem Abschluss einer Zusatzversicherung, vgl. quantitativ z.B. Burzan/Kohrs 2013), sondern die soziale Kontextualisierung und Bedingungskonstellationen dieser Handlungsstrategien zu rekonstruieren. In diesen Analysen wird der Mitte heuristisch eine eigene Art des doing life (Bahl 2018: 264) oder der Lebensführung (Müller 2016) zugeschrieben, welche etwa durch die o.g. investive Statusarbeit charakterisiert ist (Schimank 2015; Schimank et al. 2014). Dabei besteht weiterhin deutlicher Forschungsbedarf im Hinblick auf kulturelle Dimensionen der Statusreproduktion in der Mittelschicht (Schad/Burzan 2018a, b).

Wie in den vorgestellten quantitativen Untersuchungen stellen sich in qualitativen Studien ebenfalls Fragen nach der Operationalisierung von Mittelschichtszugehörigkeit und was unter Abstiegsangst, Verunsicherung oder Statussorgen überhaupt zu verstehen ist. Zur Identifizierung der Mittelschichten werden hier weniger allein ressourcenorientierte Indikatoren verwendet, sondern es wird stärker (auch) auf die Mentalitäten bzw. die habituellen Eigenschaften der Schichtzugehörigkeit geschaut (Burzan 2017). Dabei wird davon ausgegangen, dass die Ressourcen keine bestimmte Art der Lebensführung determinieren, aber trotzdem zumindest nahelegen (Schimank et al. 2014: 27). So werden etwa einzelne berufliche Milieus (z.B. in der Kreativbranche bei Koppetsch 2006; Manske 2016) untersucht oder auf der Basis der aus der Marktforschung bekannten SINUS-Milieus identifiziert (Koppetsch/Speck 2015). Tendenziell werden zudem vermehrt in Zeitdiagnosen genannte Segmente innerhalb der Mitte - z.B. die noch genauer zu untersuchende sogenannte traditionelle Mittelschicht und die postindustrielle-akademische Mittelschicht (Reckwitz 2017, Koppetsch 2018a) - fokussiert. Eine andere Variante besteht darin, historisch aus Teilen des Bürgertums und dem sog. alten und neuen Mit- 
telstand hergeleitete Berufsfelder (exemplarisch z.B. akademische Professionen oder mittelständisches Handwerk) zu identifizieren, die durch spezifische Berufsmentalitäten gekennzeichnet sind (Schad und Burzan 2018a; Hense und Schad 2019). Schimank (2018) unterscheidet in mittleren Lagen horizontal Berufsgruppen, in denen entweder das kulturelle oder das ökonomische Kapital dominiert. So besteht im Unterschied zu quantitativen Analysen hier nicht der Anspruch, Aussagen für die Großgruppe der Mittelschichtangehörigen zu treffen. Ein weiterer Unterschied ist zudem, dass nicht vorwiegend die Dimension der Ängste oder Sorgen (bezogen auf unterschiedliche Themenbereiche) abgefragt wird, sondern es werden in der Regel in offenerer Form etwaige Irritationen der bisherigen Lebensführung in einem weiteren Kontext adressiert. Dabei werden Aussagen über Verläufe und Entwicklungen in den qualitativen Untersuchungen zumeist auf der Grundlage retrospektiver Daten (wie biographischen Erzählungen) getroffen, und nicht anhand von Längsschnittdaten.

Bei der Beschreibung kultureller Charakteristika von Mittelschichtangehörigen finden sich in den bestehenden Untersuchungen wie bereits angedeutet häufig folgende Merkmale: Orientierungen wie Leistungs- und Innovationswille, ein ausgeprägter Planungsimperativ, Kaufkraft, Trägerschaft sozialer Sicherung, individuelles Aufstiegsstreben, ein Ethos der Mäßigung oder etwa eine rationale Alltagsgestaltung (vgl. u.a. Hradil/Schmidt 2007; Sachweh/Lenz 2018). Schimank et al. (2014) identifizieren insbesondere das bereits genannte Investitionskalkül als zentrales Moment der Lebensführung, welches Mittelschichtangehörige prägt. Verunsicherung oder genereller Irritationen (die nicht allein negativ konnotiert sein müssen) entstehen diesem Forschungsstrang zufolge dann, wenn die bisher gewohnte investive Statusarbeit nicht mehr so einfach möglich ist. ${ }^{12}$ So ergeben sich Konsequenzen für die Identitätsarbeit und etwa neue Abgrenzungspraktiken nach unten oder symbolische Grenzziehungen zu anderen sozialen Lagen (Sachweh/Lenz/Eicher 2018). Dabei stehen unterschiedliche (mit dem genannten doing life verbundene) Normalitätsvorstellungen selbst in Frage.

\section{Irritationen und Bewältigungsstrategien}

Qualitative Studien, die die kulturelle Dimension des Statuserhalts und des Statusverlusts in den Blick nehmen, fragen allerdings nicht nur, unter welchen Bedingungen und wie Statusbedrohungen wahrgenommen werden, sondern auch, welche Handlungsmuster bzw. Bewältigungsstrategien auf diese folgen können. Auch zu dieser Perspektive solcher mit Sicherheit oder Unsicherheit verbundenen Handlungsmustern stellen wir Befunde aus einer eigenen qualitativen Studie (Burzan et al. 2014, Burzan 2017) vor. Auf der Basis von 27 leitfadengestützten Interviews wird das Forschungsinteresse auf die Un-/Sicherheitsgefühle und erfahrungen in verschiedenen Lebensbereichen gelenkt. Dabei konzentriert sich die Studie auf erwerbstätige Mitglieder in den Mittelschichten, die bislang (trotz teilweise vorliegender erwerbsbiographischer Brüche) nicht mit einem gravierenden Abstieg konfrontiert sind. Die akademisch gebildeten und berufstätigen Befragten stammen dabei aus zwei unterschiedlichen Berufsgruppen: zum einen qualifizierte Angestellte im administrativen Bereich privater

\footnotetext{
${ }^{12}$ Dabei existieren auch Extremfälle in Bezug auf die Lage oder Mentalitäten, wenn etwa eine Privatinsolvenz angemeldet werden musste (Pfeil/Müller/Dengel 2018) oder - wie nach den Befunden von Bahl (2018) - die Sicherung der Gegenwart wichtiger wird als Investitionen in die Zukunft und gar eine Statusfatalität bei Mittelschichtangehörigen zu beobachten ist.
} 
Unternehmen, die etwa in den Bereichen Personalwesen oder Projektmanagement in fester Anstellung tätig sind, zum anderen Journalistinnen und Journalisten in Print-, Radio-, TVund Online-Medien in variierenden Beschäftigungsverhältnissen (von festangestellten Redakteuren bis zu frei Mitarbeitenden). Das Ziel der Untersuchung bestand darin, Sicherheit anstrebende oder Unsicherheit vermeidende Handlungsmuster zu identifizieren und eine Typologie zu erstellen (Burzan et al. 2014: 102).

Für die hier vorliegende Fragestellung fällt ein Befund aus der Studie ganz besonders auf: Die Einschätzung, ob die eigene berufliche Situation durch Unsicherheit geprägt sei, zum einen und die prinzipielle Bewertung von Unsicherheit als Bedrohung oder nicht zum anderen - dies sind die beiden Dimensionen, die unsere Typologie charakterisieren (Tabelle 2) - gehen nicht zwingend miteinander einher.

Tabelle 2: Typologie des Unsicherheitsempfindens und -handelns

\begin{tabular}{|l|l|l|}
\hline & $\begin{array}{l}\text { Ohne Unsicherheit im Er- } \\
\text { werbsbereich }\end{array}$ & $\begin{array}{l}\text { Mit Unsicherheit im Erwerbs- } \\
\text { bereich }\end{array}$ \\
\hline $\begin{array}{l}\text { Unsicherheit wird nicht als } \\
\text { Bedrohung empfunden }\end{array}$ & Sich sicher fühlen & Unsicherheit aushalten \\
\hline $\begin{array}{l}\text { Unsicherheit wird prinzipiell } \\
\text { als (latente) Bedrohung emp- } \\
\text { funden }\end{array}$ & $\begin{array}{l}\text { Sicherheit fortgesetzt herstel- } \\
\text { len (latente Bedrohung) } \\
\text { Unsicherheit vermeiden (ma- } \\
\text { nifeste Bedrohung) }\end{array}$ & Unsicherheit bekämpfen \\
\hline
\end{tabular}

Quelle: eigene Darstellung

Die Wahrnehmung etwaiger künftiger Diskontinuitäten oder auch Irritationen müssen also nicht bedeuten, dass aus dieser Wahrnehmung heraus Sorgen oder Statusängste entstehen. So gab es beispielsweise (im Feld rechts oben) jüngere freiberuflich tätige Journalistinnen (noch) ohne Kinder, die eine berufliche Profil- und Netzwerkbildung anstrebten, um ggf. mittel- bis langfristig eine sicherere Position zu erreichen. In der gegenwärtigen Lebensphase waren sie aber durchaus bereit, Unsicherheiten auszuhalten und lehnten z.B. eine feste Stelle ab, wenn die Inhalte und der Arbeitsort den eigenen Wünschen nicht entsprachen. Wie realistisch in die Zukunft verlagerte Sicherheitserwartungen - hier spiegelt sich die den Mittelschichten oft als typisch zugeschriebene Bereitschaft zur aufgeschobenen Bedürfnisbefriedigung - dabei angesichts der Entwicklung von Beschäftigungsverhältnissen (gerade auch im Journalismus) sind, ist kein Thema, das die Interviewten ausdrücklich ansprechen.

Umgekehrt gibt es Fälle, bei denen die Betreffenden keine relevante Unsicherheit im Erwerbsbereich zum Ausdruck bringen, die aber dennoch Unsicherheit prinzipiell als zumindest latente Bedrohung empfinden (im Feld links unten). Personen im Typus Sicherheit fortgesetzt herstellen etwa empfinden ihre berufliche Situation (zumeist als qualifizierte Angestellte in der Administration) als sicher, doch sind Aktivitäten erforderlich, um diesen Status quo auch mit Blick auf die Zukunft zu erhalten. Bei solchen Aktivitäten handelt sich beispielsweise um den 
Ausbau möglichst breit anschlussfähiger beruflicher Kompetenzen (im Unterschied zur Profilbildung bei den o.g. Journalist/innen) oder um den Statuserhalt der eigenen Kinder. So sprach ein Befragter mit seinen Kindern zu Hause Französisch, um ihnen frühzeitig Sprachkompetenzen zu vermitteln und ihnen Konkurrenzvorteile zu verschaffen. Für diesen Typus ist zudem charakteristisch, dass die Explikation von Sorgen, Unsicherheit oder auch vorsorgender Planung nicht zur Selbstdarstellung der Betreffenden gehört, sondern diese Unsicherheit abwehrenden oder vermeidenden Haltungen und Handlungen vor allem im Kontext des Gesamtinterviews zu Ausdruck kommen. So gibt es beispielsweise zwar oft Vorsorgeaktivitäten, sie werden jedoch vielfach nur auf Nachfrage oder etwas geniert erwähnt. Es ist fraglich, ob diese Personen in einer standardisierten Befragung beispielsweise angegeben hätten, sich große Sorgen um bestimmte Lebensbereiche zu machen.

Eine andere Variante der Kombination von relativer persönlicher Sicherheit im Beruf und latenter Bedrohungsempfindung durch Unsicherheit wird durch den Typus Unsicherheit vermeiden konstituiert. Obwohl die Personen, die diesen Typus bilden, mit ihrer beruflichen und/oder Lebenssituation eher unzufrieden sind, gehen sie zugunsten der ihnen wichtigen Sicherheit kein Risiko im Zuge einer Veränderung ihres Lebens ein. Nachdem sie mit Brüchen ihrer Wünsche konfrontiert wurden (z.B. mit dem Scheitern einer Ehe oder beruflich mit der Nichterreichung einer angestrebten Führungsposition), blenden sie die unwägbare Zukunft tendenziell aus.

Neben diesen Typen, bei denen ein gegenwärtiges Un-/Sicherheitsempfinden im Beruf und die Wahrnehmung einer potentiellen Bedrohung durch Unsicherheit nicht einhergehen, gab es jedoch ebenso Beispiele für diejenigen in einer etablierten beruflichen Situation, die sich auch sicher fühlen (im Feld links oben) sowie diejenigen, die der ursprünglichen Krisendiagnose entsprechen, also solche, die sich im Beruf unsicher fühlen, dies als Bedrohung empfinden und sich für eine sicherere Position einsetzen (im Feld rechts unten).

Es ist wenig überraschend: Die Mittelschichten als solche weisen keine homogene Reaktionsweisen auf sich wandelnde Bedingungen der Erwerbsarbeit auf. Ebenso wenig lassen sich einheitlich als mittelschichttypisch zugeschriebene Mentalitätselemente finden, z.B. möglichst langfristige Planungen. Dies ist wichtig zu erinnern, wenn (quantitativ) größer oder kleiner werdende Sorgen in der Mitte diagnostiziert werden. Vielmehr sind Merkmale der Lebenssituation differenzierter zu betrachten, um zu explorieren, wie und inwiefern verunsichert sich Mittelschichtangehörige fühlen und auch, wie sie solche etwaigen Sorgen nach außen (u.a. in einer Interviewsituation) darstellen. Einflussreich dafür sind unter anderem das Berufsfeld, die Lebensphase und bisherige nicht allein berufliche, sondern auch private biographische Erfahrungen.

Es zeigt sich somit, dass unsere quantitativen und qualitativen Befunde in einem Komplementärverhältnis stehen. Mit beiden Zugängen kommt eine Tendenz eines längerfristigen Sicherheitsbedürfnisses zum Ausdruck, das sich quantitativ im höheren Niveau der Sorgen um die Altersversorgung gegenüber Sorgen um die gegenwärtige wirtschaftliche Situation äußert und qualitativ dadurch verdeutlicht wird, dass ansonsten verschiedene Typen für die weitere Zukunft (mehr) Sicherheit anstreben, allerdings ohne dass dies mit einer entsprechenden Lebensplanung einhergehen muss. Stärken der quantitativen Forschung liegen dabei in der Perspektive auf breite (Schicht-)Vergleiche und in der Analyse von Antworten auf die 
gleiche Frage im Zeitverlauf. In standardisierter Form nicht geäußerte Sorgen und zumindest latente oder auf bestimmte Bereiche oder Phasen gerichtete Verunsicherungen im Leitfadeninterview schließen sich dabei nicht aus, ohne dass eines der beiden Resultate ungültig sein müsste. Mit qualitativen Daten bekommt man eine Relationierung von (nicht) geäußerten Sorgen zum einen und situativen Inszenierungen, kürzer- oder längerfristigen Bezügen und für die Einschätzung besonders relevanten Lebensbereichen zum anderen spezifischer in den Blick. ${ }^{13}$ Beispielsweise zeigen die qualitativen Ergebnisse, dass eine optimistische Perspektive auf die eigene Situation nicht bedeuten muss, dass sich auf die Zukunft gerichtete (nach wie vor zum Teil stabile) Sicherheitserwartungen (wie im Beispiel der genannten Journalistin) unter den gegebenen Bedingungen des Wandels der Erwerbsarbeit auf konkrete Grundlagen für diesen Optimismus stützen. Es sind möglicherweise Irritationen und Bewältigungsstrategien vorhanden, ohne dass explizite Sorgen verbalisiert werden. Irritationen sind also „vom Individuum wahrgenommene Störungen dessen, was es als normalen Ablauf typischer Praktiken seiner Lebensführung kennt und oftmals hochgradig habitualisiert hat" (Schimank et al. 2014: 60). Dies können grundlegende Lebenskrisen wie der Verlust des Arbeitsplatzes sein oder auch sich schleichend verdichtende Erfahrungen wie beispielsweise, dass die eigenen Kinder sich von Praktikum zu Praktikum hangeln, ohne eine feste Anstellung zu finden. Manchmal sind es aber auch nur vergleichsweise kleine Handlungsbarrieren, die ein routiniertes Handeln unmöglich machen. ${ }^{14}$ Und so kommt auch Koppetsch (2016: 237) zu dem Schluss: „Was die Akteure in der Mittelschicht beunruhigt, ist oft weniger eine akute Bedrohung oder ein plötzlicher Status- oder Einkommensverlust, sondern die zunehmende Kontingenz gesellschaftlicher Existenz- und Aufstiegsbedingungen." Dazu können u.a. Wohlstandsvorstellungen, was ein gutes Leben beinhaltet (sei es etwa ein eigenes Auto, Reisen u.ä., Schad 2017), gehören, ein Familienideal (ggf. mit einem männlichen Haupternährer, Koppetsch/Speck 2015) oder die Vorstellung, dass die eigenen Kinder einen zumindest ähnlichen Status erreichen wie die vorherige Generation. Dabei können Sorgen durchaus ein Teil von Irritationen sein, z.B. die Sorge, sich das Auto nicht mehr leisten zu können, die Sorge, dass der Familienvater seine Vollzeitstelle verliert, oder die Sorge um den Berufseinstieg der Kinder. Irritationen und Sorgen lassen sich jedoch nicht gleichsetzen. So haben wir es im qualitativen Material häufig mit ganz unterschiedlichen Inhalten zu tun, wenn der Begriff der Sorge selbst von den Interviewten verwendet wird oder ein Irritiertsein zum Ausdruck gebracht bzw.

\footnotetext{
${ }^{13}$ Interpretationsgrenzen bestehen dabei u.a. darin, dass man mit Interviewdaten keinen systematischen Vergleich dazu hat, wie Menschen sich in verschiedenen sozialen Situationen inszenieren, und Rückblicke erfolgen zwangsläufig aus der gegenwärtigen Sicht heraus. Ein theoretisch gesättigtes Spektrum von Bedingungskonstellationen - und sei es lediglich in zwei ausgewählten Berufsfeldern - stellt zudem ein Ideal dar, dem sich konkrete Studien immer nur bestmöglich annähern können.

${ }^{14}$ Klassische Störfelder sind laut Schimank et al. (2014: 60ff.) Arbeit, Partnerschaft, Wohlfahrtsstaat, Vermögensbildung, Elternschaft, politisches Engagement sowie zwei querliegende Felder: die kulturellen Orientierungen und Körperlichkeit. Drei idealtypische Handlungsoptionen für das Beispiel der Statusirritationen sind etwa (Schad/Burzan 2018b: 111): a) Ein Festhalten an bisherigen Prinzipien der Bildung und Leistung mit Tendenz zur sozialen Schließung; b) ein inkrementalistisches Coping, also ein kurzfristigeres Sich-Durchwursteln ohne grundsätzliche Umorientierung oder c) eine Neuorientierung, nach der Bastelbiographien und subjektive Unsicherheiten als Begleiterscheinung von Individualisierung zunehmend an Normalität gewinnen und die (auch in der Mittelschicht) Sicherheitserwartungen reduziert.
} 
vom Forschenden rekonstruiert wird, wie auch das folgende Beispiel einer empirischen Untersuchung zeigt.

\section{Statuserhalt in Familien}

In einem derzeit laufenden Forschungsprojekt ${ }^{15}$ untersuchen wir über etwaige individuelle Verunsicherungen hinaus, welche Rolle die Statusreproduktion auch im intergenerationalen Sinne in Familien aus verschiedenen Berufsfeldern spielt. Irritationen oder Sorgen, die auf die weitere Zukunft gerichtet sind (und für deren Relevanz auch die quantitativen Befunde sprechen), kommen auf diese Weise in den Blick. Die verbreitete Erwartung und Hoffnung, die eigenen Kinder würden später einen gleichen oder gar besseren sozialen Status erreichen, steht vor Herausforderungen: Insbesondere die Individualisierung biographischer Verläufe und der vergleichsweise späte Berufseinstieg jüngerer Generationen, Flexibilisierungs- und Prekarisierungsprozesse auf dem Erwerbsarbeitsmarkt, aber auch Veränderungen in einzelnen Branchen (etwa eine Akademisierung im Handwerk) sind solche Herausforderungen, die für den Statuserhalt in der Generationenfolge Irritationspotenzial bieten. In unserer Untersuchung betrachten wir drei Generationen und gehen angesichts des Wandels gesellschaftlicher Makrobedingungen insbesondere im Übergang von der zweiten zur dritten Generation von möglichen Verunsicherungen aus.

An einem exemplarischen Beispiel eines Interviews mit drei Generationen aus einem mittelständischen Familienunternehmen (d.h. dem sogenannten alten Mittelstand), das derzeit in der zweiten Generation durch die Angehörigen der mittleren Generation geführt wird, wird durch erste explorative Analysen deutlich, dass mit der Verwendung des Begriffs „Sorge“ im Interview u.a. ganz andere Phänomene zum Ausdruck gebracht werden als solche, die sich auf den sozialen Status oder die wirtschaftliche Situation beziehen. Sie richten sich etwa auf Sorgen um die Sicherheit der Töchter im Teenageralter, wenn diese abends ausgehen. Die Nennung einer Sorge hat hier einen konkreten Anlass und ist in der Alltagswelt verortet. Unsicherheiten um die vergleichsweise abstrakte und in der Zukunft liegende Nachfolgeregelung für den eigenen Familienbetrieb werden demgegenüber weniger im semantischen Rahmen einer Sorge thematisiert, sind aber gleichwohl virulent. ${ }^{16}$. Die beiden Töchter der jungen Generation, 18 und 21 Jahre alt, streben keine für den Betrieb im engeren Sinne einschlägige berufliche Laufbahn an. Die Nachfolgefrage birgt damit ein Unsicherheitspotenzial sowohl für die Eltern als auch für die Kindergeneration: Die Eltern, Mitte 50, stehen vor der Entscheidung, den Betrieb - zumal in Zeiten eines raschen technologischen Wandels - zu einem möglichst günstigen Zeitpunkt zu verkaufen, um ihre eigene Altersversorgung zu sichern, sofern sich nicht eine der Töchter doch noch für eine Betriebsübernahme entscheidet. Die Töchter der jüngeren Generation befinden sich unter Bedingungen nicht zuletzt dieses Zeitdrucks vor der Entscheidung, möglichst bald für oder gegen die Betriebsübernahme zu votieren. Im Falle des Verkaufs des Unternehmens ist im Grunde unklar, ob die Töchter - die eine hat gerade

\footnotetext{
${ }^{15}$ Das Projekt „Statuserhalt in der `sozialen Mitte‘. Intergenerationale Stabilisierungsmechanismen in Berufsfeldern der Mittelschicht“ in Kooperation mit dem SOFI Göttingen (Berthold Vogel/Andrea Hense) wird von 2018 bis 2020 von der DFG gefördert (vgl. auch Schad/Burzan 2018a, 2018b).

${ }^{16}$ Dieser Befund birgt somit wiederum Reflexionsanregungen für die Aussagekraft von in Surveys geäußerten Sorgen.
} 
eine Ausbildung im Verwaltungsbereich abgeschlossen, die andere strebt eine Lehre nach dem bevorstehenden Abitur an - den Status der Eltern und Großeltern als mittelständische Unternehmer aufrechterhalten können. Obwohl die Angehörigen der mittleren Generation betonen, anders als der eigene Vater keinen autoritären Druck ausüben zu wollen und auch zu sehen, dass Unsicherheiten über den weiteren beruflichen Weg nach dem Schulabschluss heutzutage normal seien, zeigt sich die Verunsicherung recht deutlich, und zwar ohne dass Sorgen oder Ängste explizit angesprochen würden. Stattdessen werden sowohl Pro- als auch Contra-Argumente für die Übernahme durch die Elterngeneration an verschiedenen Stellen im Interview geäußert und signalisieren dadurch einen Konflikt. Der Vater sagt etwa an einer Stelle im Hinblick auf die Nachfolgefrage:

Die [Töchter] sollen das selbst entscheiden, die sind dann irgendwann alt genug. $\mathrm{Ob}$ das der richtige Weg ist, müssen sie dann auch selbst entscheiden. Aber die sind ja dann selbst für sich im Prinzip verantwortlich oder die sollen ihren eigenen Weg dann auch gehen. Ob der dann immer richtig ist, wird sich ja dann auch zeigen. Aber ich wollte nie, dass die dann von mir das irgendwie vordiktiert kriegen. Natürlich wär' das schön, wenn hier jemand mit einsteigt, denn wir werden ja auch nicht jünger.

Später heißt es dann im Kontext u.a. technologischer Anpassungserfordernisse:

Wir [sagen] jetzt auch schon: ,Mh, wer weiß, was in zehn Jahren mal ist?' Da müssten die Kinder eigentlich verrückt sein, wenn die in so ein Unternehmen einsteigen würden, mit der ganzen Last und der ganzen Verantwortung, die man hat.

Diese Verunsicherung zeigt sich auch bei seiner Tochter: Auf die Frage der Interviewerin, ob es sie reizen könne, auch als Selbständige im Familienbetrieb tätig zu werden, antwortet sie:

Ich weiß nicht so. Also kann ich mir jetzt irgendwie noch nicht so-- vorstellen, muss ich sagen. Also- weil das ist so viel, was man dann-also klar, das ist alles hier so aufgebaut schon, aber das so alleine wei- also ich weiß ja nicht, was meine Schwester, wenn meine Schwester sagt, sie will das halt nicht - alleine, als einzige Person dann, das funktioniert ja gar nicht. Also dann müsste man halt wieder gucken, dass man Mitarbeiter hat. Gut, wer weiß, wie lange meine Eltern, meine Tante, ne? Weiß man ja auch nicht, wie lange das halt alles so funktioniert. Also ich weiß nicht. Kann ich mir irgendwie nicht so [lacht] nicht so vorstellen.

Daraufhin ergänzt der Vater: „Müsste dann schon irgendwie einen Partner mitbringen.“ Worauf die Tochter antwortet: „Genau. Genau.“ Und die Mutter steuert bei: „Musst du einen kennenlernen.“ Daraufhin weist die Tochter auf die notwendige Passung des potenziellen Partners hin: „Müsste ich jemanden kennenlernen, der halt dann so-“, und die Mutter unterbricht scherzhaft: „Stellenausschreibung, hätte ich fast gesagt! [lacht]“. So greifen sowohl die Tochter als auch die Eltern im Kontext der zunehmenden Kontingenz berufsbiographischer 
Verläufe und normativ postulierter Wahlfreiheit bei der Berufsfindung (zumindest gedanklich) auf etablierte und in der Familie über mindestens zwei Generationen praktizierte geschlechtsspezifische Vorstellungen von Arbeitsteilung innerhalb des Betriebs zurück und identifizieren damit im Gespräch einen potenziellen Stabilisierungsanker als mögliches zukünftiges Szenario - für dessen Realisierung aber noch die entsprechenden Bedingungen geschaffen werden müssten. Natürlich ist bei der Deutung dieser Aussagen auch die Interviewsituation zu berücksichtigen. Möglicherweise fordert die Interviewerin hier zu einer Explikation von Fragen auf, deren konkrete Thematisierung im Familienkontext bislang eher vermieden wurde, was die Unsicherheit situativ verstärkt. Die Option, ein geeigneter Schwiegersohn könne die Lösung des Nachfolgeproblems sein, verschiebt die anstehende Nachfolgeentscheidung jedenfalls in die mittlere Zukunft, was zugleich jedoch die Unsicherheitssituation auf Dauer stellt.

Für die vorliegende Frage nach der methodischen Erfassung von Irritationen und Unsicherheiten sei noch einmal betont, dass die Familie (betrachtet man den Gesamtkontext des Interviews) kaum dezidierte Statussorgen äußert, sondern sich Irritationen zum Teil erst aus der Rekonstruktion des Gesagten im Zusammenhang ergeben. So gibt es in dieser Familie einerseits die Nachfolgefrage, aber für die älteren Generationen ist der Status der mittelständischen Unternehmer andererseits sehr selbstverständlich, und zumindest teilweise sehen sie Unwägbarkeiten nicht als spezifisches Problem ihrer Familie, sondern als gewisse Normalität, mit denen auch andere umgehen müssen, ${ }^{17}$ was die damit potenziell einhergehenden Ängste tendenziell mindert. Auch dieses empirische, hier zunächst explorativ genutzte Beispiel verweist also darauf, dass statusbezogene Unsicherheiten und deren Inszenierung stark variierende Ausdrucksformen aufweisen, was methodische Herausforderungen mit sich bringt.

Es lässt sich somit erneut zeigen, dass innerhalb der Mittelschichten unterschiedliche Segmente bestehen, deren Differenzierung bis heute empirisch nicht ausreichend erforscht und beschrieben ist. Die Ausdrucksformen von Unsicherheit können in diesen Segmenten variieren und in Ausmaß und Intensität divergent sein. In der Forschung finden sich vor allem Befunde zu groben Unterscheidungen wie der oberen, mittleren und unteren Mittelschicht, die in statistischen Analysen und etwa der breiten Literatur zur upper (z.B. Lamont 1992; Andreotti/Le Galès/Moreno Fuentes 2015) bzw. lower middle class (z.B. Felski 2000) ihren Niederschlag finden. So beobachten Sachweh und Lenz (2018) z.B. auf der Basis von Gruppendiskussionen eine recht hohe sozioökonomische Zufriedenheit in der unteren Mitte, die auf dem bereits erwähnten Ethos der Mäßigung und des planvollen Realismus basiert. Dabei wurden die Teilnehmenden der Gruppendiskussion auf der Basis ihres Bildungsabschlusses und aktuellen Berufs (etwa als Sachbearbeiter/in oder Pflegekraft) zugeordnet. Befunde zu stärker an kulturelle Hegemoniekonzepte rückgebundene Unterscheidungen zwischen traditioneller und postindustriell-akademischer Mittelschicht (Reckwitz 2017) stehen noch aus. Daher muss je nach Schichtsegment genauer gefragt werden, wie hier Sorgen wahrgenommen, geäußert oder verhandelt werden. So kann es etwa einen zentralen Unterschied für die Unsicherheitswahrnehmung und -bewältigung sowie für Identitätskonstruktionen innerhalb der Mittelschicht machen, ob Wohneigentum in der Familie besteht oder realisier-

${ }^{17}$ Z.B. im Hinblick auf Effekte des technologischen Wandels oder Trial-and-Error-Haltungen bei der Wahl von Berufswegen in der jungen Generation. 
bar erscheint (Benson/Jackson 2017). Es lässt sich sogar annehmen, dass sich neue Konstellationen und Annäherungen oder Spaltungen innerhalb mittlerer sozialer Lagen durch etwaige Verunsicherungen oder Irritationen ergeben. So zeichnet sich in der US-amerikanischen oder in europäischen Gesellschaften im Kontext des verstärkt auftretenden Rechtspopulismus ein Konfliktfeld an den Rändern und innerhalb der Mittelschicht ab, in welchem etwa auf der Basis von Othering (Wuthnow 2017) neue Gräben und Allianzen insbesondere gegen so genannte politische Eliten entstehen. Durch solche und andere Dynamiken nehmen entsprechend auch die Herausforderungen empirischer Forschung zu, die bei der Exploration heterogener und sich ausdifferenzierender mittlerer Lagen bestehen. Auch für die quantitative Forschung ist entsprechend darüber nachzudenken, welche Indikatoren über klassische Merkmale wie Einkommen, Beruf oder Bildung hinaus erfasst werden müssten, um aussagekräftige Lagesegmente zu identifizieren.

\section{Fazit}

Die empirische Erforschung und darauf basierende gesellschaftsdiagnostische Bewertung potentieller Erosionstendenzen in den Mittelschichten ist ein herausforderndes Unterfangen weil erstens unterschiedliche Operationalisierungen unter Berücksichtigung von verschiedenen Ressourcen und Mentalitätselementen zu unterschiedlichen Befunden sowie deren Interpretation führen können, weil es zweitens die (sowohl vertikal als auch horizontal differenzierten) Mittelschichten als vergleichsweise homogene Gruppierung nicht gibt und weil man drittens mit quantitativen und qualitativen Methoden unterschiedliche Perspektiven auf den Forschungsgegenstand einnimmt, deren Verknüpfung sorgfältig zu reflektieren ist (Burzan 2016).

Entsprechend geraten Forschende in eine defensive Position, wenn sie (etwa in den ersten Jahren der 2000er Jahre) erklären mussten, warum Verunsicherungen in den Mittelschichten oft höher waren als konkrete Abstiegsrisiken, oder wenn sie mit dem (ggf. scheinbaren) Widerspruch konfrontiert sind, dass geäußerte Verunsicherungen zwar rückläufig sind, es aber zugleich Hinweise dafür gibt, dass Statuskonkurrenzen und soziale Schließungen zunehmen was nicht zuletzt für (sozial)politische Schlussfolgerungen relevant ist.

Mit unseren Ausführungen zum Forschungsstand und zu eigenen empirischen Befunden zu den Mittelschichten in Deutschland haben wir gezeigt, dass zum einen bestimmte Tendenzen auch dann bestehen, wenn z.B. Schichtzuordnungen oder Verunsicherungen unterschiedlich operationalisiert werden: So gingen geäußerte Sorgen in den letzten Jahren zurück. Zum anderen ist jedoch von deutlichen Differenzierungen innerhalb der Mittelschichten auszugehen, die keinesfalls allein über eine verfeinerte Einkommensuntergliederung erfassbar sind, sondern andere Ressourcen (wie Bildung und Beruf) ebenso betreffen wie horizontale (Mentalitäts-)Unterschiede. In den verschiedenen Segmenten sind nicht allein z.B. Orientierungen unterschiedlich, sondern auch, wie man etwaige Verunsicherungen nach außen in welchen Kontexten inszeniert und wie sich in der Handlungspraxis z.B. spezifische Schließungstendenzen vollziehen. Insbesondere die Perspektive auf den Zusammenhang sozioökonomischer und kultureller Dimensionen sozialer Ungleichheit kann somit erhellen, dass etwa vergleichsweise geringe akute wirtschaftliche Sorgen einerseits und Statusängste (etwa aufgrund latenter 
Empfindungen der Infragestellung kultureller Hegemonien) andererseits miteinander einhergehen können. Die Verknüpfung quantitativer und qualitativer Methoden kann zur Erforschung dieser Fragen einen wichtigen Beitrag leisten: quantitative Methoden durch eine Überblicksperspektive im Zeit- und Schichtvergleich (unter Nutzung von Datensätzen mit hohen Fallzahlen), qualitative Methoden durch einen differenzierten Blick auf spezifische Segmente der Mittelschichten und dort auf die Wahrnehmung sozialer Lagen, ungleichheitsrelevante Orientierungen und Handlungsweisen in ihrer komplexen Verwobenheit (vgl. auch Burzan/Schad 2018). Am konkreten Beispiel lässt sich jedoch auch zeigen, dass es keine reibungslose Komplementarität zwischen beiden Forschungssträngen mit ihren unterschiedlichen Forschungslogiken gibt, sondern dass z.B. ein je spezifisches Verständnis von Verunsicherung eine direkte Vergleichbarkeit erschwert. In beiden Fällen gilt: So wenig, wie ein heuristischer Anknüpfungspunkt bei möglichen Erosionen in der Mitte den Blick dafür verstellen sollte, dass es (je nachdem, wen man (nicht) zur Mitte zählt) nicht überall nur Krisen zu sehen gibt, so wenig sollte umgekehrt eine Offenheit dafür fehlen, dass (z.B. im Interview) nicht als solche deklarierte Unsicherheiten bereits bedeuten, dass Abgrenzungskämpfe und Statusreproduktionsstrategien nicht existierten. Zu den Aufgaben weiterer Forschungen zur Lage der Mittelschichten gehört es, methodisch sorgfältig zu reflektieren, welche Perspektiven man jeweils mit spezifischen Forschungskonzepten und Erhebungsinstrumenten (auch über Befragungen hinaus) einnimmt. Zudem gilt es zu erforschen, welche Dynamiken des Zusammenspiels sozialer Lagen und kultureller Orientierungen für vertikal und horizontal angemessen differenzierte Gruppierungen unter Berücksichtigung gerade auch von Ambivalenzen ${ }^{18}$ existieren. Einzelne Befunde mit je spezifischer Perspektive und Reichweite sind daher durch verschränkte (triangulierende) Perspektiven zu ergänzen, mit Hilfe derer anstelle vorschneller Schlussfolgerungen in Bezug auf eine weitreichende Erosion mittlerer Lagen oder umfassende Entwarnungen differenzierte Diagnosen ermöglicht werden.

\section{Literatur}

Amoranto, Glenita, Natalie Chun und Anil Deolalikar (2010). Who Are the Middle Class and What Values Do They Hold? Evidence from the World Values Survey. Asian Development Economics Working Paper Series No. 229.

Andreotti, Alberta, Patrick Le Galès und Francisco Javier Moreno Fuentes (2015). Globalised Minds, Roots in the City. Urban Upper-Middle Classes in Europe. Studies in Urban and Social Change. Chichester: John Wiley \& Sons Inc.

Arndt, Christian (2015). Das Vermögen der mittleren Einkommensschicht in Deutschland zu Beginn des 21. Jahrhunderts. Was wissen wir über die Mittelschicht in Deutschland? Berlin: Konrad-AdenauerStiftung.

\footnotetext{
${ }^{18}$ Hinsichtlich der sozialen Lage ist hier beispielsweise an Angehörige des sogenannten akademischen Prekariats zu denken (Sander 2012), die hohe Bildungsressourcen aufweisen, die allerdings z.B. länger in befristeten Beschäftigungen (etwa in der Wissenschaft) tätig sind, ggf. auch Phasen ohne Erwerbstätigkeit aufweisen und die damit in Bezug auf ihre Einnahmen und Lebenssituation kaum längerfristig planen können.
} 
Bahl, Friederike (2018). Über Proletarität und Abgrenzungspraktiken. Beobachtungen im unteren Dienstleistungssegment. In: Schöneck, Nadine M. und Sabine Ritter (Hg.). Die Mitte als Kampfzone. Wertorientierungen und Abgrenzungspraktiken der Mittelschichten. Bielefeld: transcript, 261276.

Benson, Michaela und Emma Jackson (2017). Making the Middle Classes on Shifting Ground? Residential Status, Performativity and Middle-Class Subjectivities in Contemporary London. The British Journal of Sociology 68(2), 215-233.

Birdsall, Nancy (2015). Does the Rise of the Middle Class Lock in Good Government in the Developing World? Essay. Center for Global Development.

Böhnke, Petra (2005). Teilhabechancen und Ausgrenzungsrisiken in Deutschland. Aus Politik und Zeitgeschichte 37: 31-36.

Bolte, Karl Martin, Dieter Kappe und Friedhelm Neidhardt (1967). Soziale Schichtung der Bundesrepublik Deutschland. In: Bolte, Karl Martin (Hg.). Deutsche Gesellschaft im Wandel. Opladen: Leske + Budrich, 233-351.

Bourdieu, Pierre (1982). Die feinen Unterschiede. Kritik der gesellschaftlichen Urteilskraft. Frankfurt a.M.: Suhrkamp.

Bude, Heinz (2011). Bildungspanik. Was unsere Gesellschaft spaltet. München: Hanser.

Bundesagentur für Arbeit (2017). Arbeitslosigkeit im Zeitverlauf. https://www.destatis.de/DE/ZahlenFakten/Indikatoren/LangeReihen/Arbeitsmarkt/lrarb003.html. (Zugriff am 19.11.2018)

Burkhardt, Christoph, Markus M. Grabka, Olaf Groh-Samberg, Yvonne Lott und Steffen Mau (2013). Mittelschicht unter Druck? Gütersloh: Bertelsmann Stiftung.

Burzan, Nicole (2016). Methodenplurale Forschung. Chancen und Probleme von Mixed Methods. Weinheim/Basel: Beltz Juventa.

Burzan, Nicole (2017). Zur intergenerationalen Stabilisierung der Zugehörigkeit zur Mittelschicht. In: Lessenich, Stephan (Hg.). Geschlossene Gesellschaften. Verhandlungen des 38. Kongresses der Deutschen Gesellschaft für Soziologie in Bamberg 2016.

http://publikationen.soziologie.de/index.php/kongressband 2016/article/view/436 (Zugriff am 03.12.2018)

Burzan, Nicole und Silke Kohrs (2013). Vielfältige Verunsicherungen in der Mittelschicht - eine Herausforderung für sozialen Zusammenhalt? In: Pries, Ludger (Hg.). Zusammenhalt durch Vielfalt? Bindungskräfte der Vergesellschaftung im 21. Jahrhundert. Wiesbaden: Springer VS, 101-119.

Burzan, Nicole, Silke Kohrs und Ivonne Küsters (2014). Die Mitte der Gesellschaft: Sicherer als erwartet? Weinheim/Basel: Beltz Juventa.

Burzan, Nicole und Miriam Schad (2018). Qualitative Ungleichheitsforschung. Zugänge zu einem Kernthema der Soziologie am Beispiel deutschsprachiger Zeitschriftenbeiträge. Zeitschrift für Qualitative Forschung 1-2, 13-29.

Castel, Robert und Klaus Dörre (Hg.) (2009). Prekarität, Abstieg, Ausgrenzung. Die soziale Frage am Beginn des 21. Jahrhunderts. Frankfurt a.M.: Campus.

Datenreport (2018). Ein Sozialbericht für die Bundesrepublik Deutschland. Statistisches Bundesamt, Wiesbaden und Wissenschaftszentrum Berlin für Sozialforschung. 
Davis, Diane E. (2004). Discipline and Development. Middle Classes and Prosperity in East Asia and Latin America. Cambridge: Cambridge University Press.

Decker, Oliver, Johannes Kiess und Elmar Brähler (Hg.) (2016). Die enthemmte Mitte. Autoritäre und rechtsextreme Einstellung in Deutschland: die Leipziger Mitte-Studie 2016. Gießen: PsychosozialVerlag.

Delhey, Jan (2012). Gleichheit fühlt sich besser an: Statusunbehagen und Wohlbefinden in europäischen Gesellschaften. Informationsdienst Soziale Indikatoren 47, 8-11.

Delhey, Jan und Georgi Dragolov (2014). Why Inequality Makes Europeans Less Happy: The Role of Distrust, Status Anxiety, and Perceived Conflict. European Sociological Review 30(2), 151-165.

DIW SOEP-Personenfragebogen (2016).

http://panel.gsoep.de/soep-docs/surveypapers/diw ssp0345.pdf (Zugriff am 19.11.2018)

Dörre, Klaus (2009). Ende der Planbarkeit? Lebensentwürfe in unsicheren Zeiten. Aus Politik und Zeitgeschichte 41: 19-24.

Erikson, Robert und John H. Goldthorpe (1992). The Constant Flux. A Study of Class Mobility in Industrial Societies. Oxford: Clarendon Press.

Felski, Rita (2000). Nothing to Declare: Identity, Shame, and the Lower Middle Class. PMLA 115(1), $33-45$.

Geiger, Theodor (1967). Die soziale Schichtung des Deutschen Volkes: Soziographischer Versuch auf statistischer Grundlage. Stuttgart: Lucius \& Lucius.

Goebel, Jan, Martin Gornig und Hartmut Häußermann (2010). Polarisierung der Einkommen: die Mittelschicht verliert. DIW-Wochenbericht 77(24), 2-8.

Grabka, Markus M. und Joachim R. Frick (2008). Schrumpfende Mittelschicht - Anzeichen einer dauerhaften Polarisierung der verfügbaren Einkommen? DIW-Wochenbericht 75(10), 101-108.

Grabka, Markus M., Jan Goebel, Carsten Schröder und Jürgen Schupp (2016). Schrumpfender Anteil an BezieherInnen mittlerer Einkommen in den USA und Deutschland. DIW Wochenbericht 83(18), 391-402.

Grabka, Markus M. und Christian Westermeier (2014). Anhaltend hohe Vermögensungleichheit in Deutschland. DIW-Wochenbericht 81(9), 151-164.

Groh-Samberg, Olaf, Steffen Mau und Uwe Schimank (2014). Investieren in den Status: Der voraussetzungsvolle Lebensführungsmodus der Mittelschichten. In: Leviathan. Berliner Zeitschrift für Sozialwissenschaft 42(2), 219-248.

Groh-Samberg, Olaf, Nepomuk Hurch und Nora Waitkus (2018). Statuskonkurrenzen und soziale Spaltungen. Zur Dynamik sozialer Ungleichheiten. WSI Mitteilungen 71(5), 347-357.

Hartmann, Michael (2018). Die Abgehobenen. Wie die Eliten die Demokratie gefährden. Frankfurt a.M.: Campus.

Hense, Andrea und Miriam Schad (2019, i.E.). Sampling von Familien in der Mittelschicht. In: Nicole Burzan (Hg.), Komplexe Dynamiken globaler und lokaler Entwicklungen. Verhandlungen des 39. Kongresses der Deutschen Gesellschaft für Soziologie in Göttingen 2018.

Hochschild, Arlie Russell (2016). Strangers in their own land. Anger and mourning on the American right. New York: The New Press. 
Horn, Gustav A., Jan Behringer, Sebastian Gechert, Katja Rietzler und Ulrike Stein (2017). Was tun gegen die Ungleichheit? Wirtschaftspolitische Vorschläge für eine reduzierte Ungleichheit, IMK Report 129, Düsseldorf.

Hradil, Stefan und Holger Schmidt (2007). Angst und Chancen. Zur Lage der gesellschaftlichen Mitte aus soziologischer Sicht. In: Herbert Quandt-Stiftung (Hg.). Zwischen Erosion und Erneuerung. Die gesellschaftliche Mitte in Deutschland: ein Lagebericht. Frankfurt a.M.: Societäts-Verlag, 163-226.

Inglehart, Ronald F und Pippa Norris (2016). Trump, Brexit, and the Rise of Populism: Economic Have-Nots and Cultural Backlash. HKS Working Paper No. RWP16-026.

Johnston, Robert D. (2013). The Radical Middle Class. Populist Democracy and the Question of Capitalism in Progressive Era Portland, Oregon. Politics and Society in Twentieth-Century America. Princeton: Princeton University Press.

Kochhar, Rakesh (2017). Middle Class Fortunes in Western Europe. http://www.pewglobal.org/2017/04/24/middle-class-fortunes-in-western-europe/ (Zugriff am 03.12.2018)

Koppetsch, Cornelia (2006). Das Ethos der Kreativen. Eine Studie zum Wandel von Arbeit und Identität am Beispiel der Werbeberufe. Konstanz: UVK.

Koppetsch, Cornelia (2016). Verunsicherungen in der Mitte der Gesellschaft. Milieuspezifische Strategien der Bewältigung sozialer Gefährdungen. In: Alleweldt, Erika, Anja Röcke und Jochen Steinbicker (Hg.). Lebensführung heute. Klasse, Bildung, Individualität. Weinheim: Beltz Juventa, 236259.

Koppetsch, Cornelia (2018a). Kosmopolitische Heimat. Räumliche Selbstvergewisserung im Brennglas transnationaler Ungleichheitskonflikte. In: Schöneck, Nadine M. und Sabine Ritter (Hg.). Die Mitte als Kampfzone. Wertorientierungen und Abgrenzungspraktiken der Mittelschichten. Bielefeld: transcript, 179-196.

Koppetsch, Cornelia (2018b). Rechtspopulismus als Klassenkampf ? Soziale Deklassierung und politische Mobilisierung. WSI Mitteilungen 71(5), 382-391.

Koppetsch, Cornelia und Sarah Speck (2015). Wenn der Mann kein Ernährer mehr ist. Geschlechterkonflikte in Krisenzeiten. Berlin: Suhrkamp.

Kraemer, Klaus (2010). Abstiegsängste in Wohlstandslagen. In: Burzan, Nicole und Peter A. Berger (Hg.). Dynamiken (in) der gesellschaftlichen Mitte. Wiesbaden: VS, 201-229.

Kriesi, Hanspeter; Edgar Grande, Romain Lachat, Martin Dolezal, Simon Bornschier und Timotheos Frey (2006). Globalization and the transformation of the national political space: Six European countries compared. European Journal of Political Research 45 (6), 921-956.

Kroeker, Lena, David O'Kane und Tabea Scharrer (Hg.) (2018). Middle Classes in Africa. Changing Lives and Conceptual Challenges. Frontiers of Globalization. Cham: Springer International Publishing.

Laatz, Wilfried (2011). Statusangst. In: Fuchs-Heinritz, Werner (Hg.). Lexikon zur Soziologie. Wiesbaden: Springer VS, 654.

Lamont, Michèle (1992). Money, Morals, and Manners. The Culture of the French and American Upper-Middle Class. Chicago: University of Chicago Press. 
Larsen, Christian Albrekt (2013). The Rise and Fall of Social Cohesion. The Construction and Deconstruction of Social Trust in the US, UK, Sweden and Denmark. Oxford: Oxford University Press.

Lengfeld, Holger und Jochen Hirschle (2009). Die Angst der Mittelschicht vor dem sozialen Abstieg. Eine Längsschnittanalyse 1984-2007. Zeitschrift für Soziologie 38(5): 379-398.

Lengfeld, Holger und Jessica Ordemann (2016). Die Angst der Mittelschicht vor dem sozialen Abstieg revisited. Eine Längsschnittanalyse 1984-2014. DIW-SOEPpapers 862, Berlin.

Lengfeld, Holger und Jessica Ordemann (2017). Der Fall der Abstiegsangst, oder: Die mittlere Mittelschicht als sensibles Zentrum der Gesellschaft. Eine Trendanalyse 1984-2014. Zeitschrift für Soziologie 46(3): 167-184.

Lengfeld, Holger und Jessica Ordemann (2018). Statuspanik in der Mittelschicht ? Aktuelle Befunde aus der Einstellungsforschung. In: Schöneck, Nadine M. und Sabine Ritter (Hg.). Die Mitte als Kampfzone. Wertorientierungen und Abgrenzungspraktiken der Mittelschichten. Bielefeld: transcript, 69-84.

Littrell, Jill, Fred Brooks, Jan Ivery und Mary Ohmer (2010). Why You Should Care About the Threatened Middle Class. Journal of Sociology \& Social Welfare 37(3), 85-112.

Manske, Alexandra (2016). Kapitalistische Geister in der Kultur- und Kreativwirtschaft. Kreative zwischen wirtschaftlichem Zwang und künstlerischem Drang. Bielefeld: transcript.

Mau, Steffen (2012). Lebenschancen. Wohin driftet die Mittelschicht? Berlin: Suhrkamp.

Milanović, Branko (2016). Global Inequality. Cambridge: The Belknap Press of Harvard University Press.

Müller, Hans-Peter (2016). Wozu Lebensführung? Eine forschungsprogrammatische Skizze im Anschluss an Max Weber. In: Alleweldt, Erika, Anja Röcke und Steinbicker (Hg.). Lebensführung heute. Klasse, Bildung, Individualität. Wirtschaft, Gesellschaft und Lebensführung. Weinheim/Basel: Beltz Juventa, 23-52.

Nachtwey, Oliver (2016). Die Abstiegsgesellschaft. Über das Aufbegehren in der regressiven Moderne. Berlin: Suhrkamp.

Niehues, Judith, Thilo Schaefer und Christoph Schröder (2013). Arm und Reich in Deutschland: Wo bleibt die Mitte? IW-Analysen 89. Köln: IW Medien.

Niehues, Judith und Anja K. Orth (2018). Die gespaltene Mitte. Werte, Einstellungen und Sorgen. RHIDiskussion 30. München: Roman-Herzog-Institut.

Parkinson, Frank (1968). Middle Class Radicalism. The Social Bases of the British Campaign for Nuclear Disarmament. Manchester: Manchester Univ. Press.

Pfeil, Patricia, Marion Müller und Udo Dengel (2018). Wer bin ich oder wo bin ich? Identitätsarbeit Mittelschichtangehöriger in Insolvenz. In: Schöneck, Nadine M. und Sabine Ritter (Hg.). Die Mitte als Kampfzone. Wertorientierungen und Abgrenzungspraktiken der Mittelschichten. Bielefeld: transcript, 277-294.

Popp, Silvia (2015). Die neue globale Mittelschicht. In: Bundeszentrale für Politische Bildung (Hg.): Oben - Mitte - Unten. Zur Vermessung der Gesellschaft. Bonn: bpb, 174-185.

Reckwitz, Andreas (2017). Die Gesellschaft der Singularitäten. Zum Strukturwandel der Moderne. Berlin: Suhrkamp. 
Sachweh, Patrick und Sarah Lenz (2018). „Maß und Mitte“ - Symbolische Grenzziehungen in der unteren Mittelschicht. Kölner Zeitschrift für Soziologie und Sozialpsychologie 70(3), 361-389.

Sander, Nadine (2012). Das akademische Prekariat. Leben zwischen Frist und Plan. Konstanz: UVK.

Schad, Miriam (2017). Über Luxus und Verzicht. Umweltaffinität und umweltrelevante Alltagspraxis in prekären Lebenslagen. München: oekom.

Schad, Miriam und Nicole Burzan (2018a). Von Generation zu Generation. Strategien des Statuserhalts im Kontext von Familien- und Berufsmentalitäten in der Mittelschicht. In: Schöneck, Nadine M. und Sabine Ritter (Hg.). Die Mitte als Kampfzone. Wertorientierungen und Abgrenzungspraktiken der Mittelschichten. Bielefeld: transcript, 109-123.

Schad, Miriam und Nicole Burzan (2018b). Intergenerationale Statusstabilisierung in der Mittelschicht - eine exemplarische Analyse zweier Unternehmensfamilien. WestEnd - Neue Zeitschrift für Sozialforschung 15 (1), 99-108.

Schimank, Uwe (2015). Lebensplanung!? Biografische Entscheidungspraktiken irritierter Mittelschichten. Berliner Journal für Soziologie 25: 7-31.

Schimank, Uwe (2018). Rechtspopulistische Mittelschichten als Gefährder gesellschaftlicher Ordnung. Eine theoretische Skizze. In: Schöneck, Nadine M. und Sabine Ritter (Hg.). Die Mitte als Kampfzone. Wertorientierungen und Abgrenzungspraktiken der Mittelschichten. Bielefeld: transcript, 217242.

Schimank, Uwe, Steffen Mau und Olaf Groh-Samberg (2014). Statusarbeit unter Druck? Zur Lebensführung der Mittelschichten. Weinheim: Beltz Juventa.

Schöneck, Nadine M., Steffen Mau und Jürgen Schupp (2011). Gefühlte Unsicherheit: Deprivationsängste und Abstiegssorgen der Bevölkerung in Deutschland. SOEP-papers Nr. 428.

Schöneck, Nadine M. und Sabine Ritter (2018). Zur Einleitung. Die gesellschaftliche Mitte als Kampfzone - Perspektiven und Fragestellungen. In: Schöneck, Nadine M. und Sabine Ritter (Hg.). Die Mitte als Kampfzone. Wertorientierungen und Abgrenzungspraktiken der Mittelschichten. Bielefeld: transcript, 11-28.

Verwiebe, Roland (2014). Die Auflösung der Mittelschicht und wachsende Armut in Deutschland. Sozialer Fortschritt, 63(12), 293-300.

Vester, Michael (2017). Der Kampf um soziale Gerechtigkeit. Der Rechtspopulismus und die Potentiale politscher Mobilisierung. http://nrw.rosalux.de/fileadmin/ls_nrw/dokumente/Publikationen/Michael_Vester_Rechtspopulis mus soziale Gerechtigkeit 18 M\%C3\%A4rz-2.pdf (Zugriff am 03.12.2018)

Vester, Michael, Peter von Oertzen, Heiko Geiling, Thomas Hermann und Dagmar Müller (2001). Soziale Milieus im gesellschaftlichen Strukturwandel. Zwischen Integration und Ausgrenzung. Frankfurt a.M.: Suhrkamp.

Vogel, Berthold (2009). Wohlstandskonflikte. Soziale Fragen, die aus der Mitte kommen. Hamburg: Hamburger Edition.

Wagner, Gert G., Joachim R. Frick und Jürgen Schupp (2007). The German Socio-Economic Panel Study (SOEP) - Scope, Evolution and Enhancements. Schmollers Jahrbuch 127, Berlin: 139-169.

Weiß, Anja (2017). Soziologie globaler Ungleichheiten. Berlin: Suhrkamp. 
Werding, Martin (2011). Mittelschicht. Sind die gefühlten Bedrohungen messbar? Wirtschaftsdienst, 91(8), 515-518.

Wilkinson, Richard G. und Kate Pickett (2010). The Spirit Level. Why Equality Is Better for Everyone; [with a New Chapter Responding to Their Critics]. London: Pinguin Sociology.

Wuthnow, Robert (2017). American Misfits and the Making of Middle-Class Respectability. Princeton: Princeton University Press.

Zick, Andreas, Beate Küpper, Daniela Krause und Wilhelm Berghan (Hg.) (2016). Gespaltene Mitte feindselige Zustände. Rechtsextreme Einstellungen in Deutschland 2016 (hrsg. für die FriedrichEbert-Stiftung von Ralf Melzer). Bonn: Dietz. 